\title{
Nonlinear Dynamic Behavior Analysis of Pressure Thin-Wall Pipe Segment with Supported Clearance at Both Ends
}

\author{
Chaofeng Li, Qiansheng Tang, and Bangchun Wen \\ School of Mechanical Engineering \& Automation, Northeastern University, Shenyang, Liaoning 110819, China \\ Correspondence should be addressed to Chaofeng Li; chaof.li@gmail.com
}

Received 24 March 2016; Revised 30 May 2016; Accepted 31 May 2016

Academic Editor: Francesco Tornabene

Copyright (C) 2016 Chaofeng Li et al. This is an open access article distributed under the Creative Commons Attribution License, which permits unrestricted use, distribution, and reproduction in any medium, provided the original work is properly cited.

\begin{abstract}
An analysis of nonlinear behaviors of pressure thin-wall pipe segment with supported clearance at both ends was presented in this paper. The model of pressure thin-wall pipe segment with supported clearance was established by assuming the restraint condition as the work of springs in the deformation directions. Based on Sanders shell theory, Galerkin method was utilized to discretize the energy equations, external excitation, and nonlinear restraint forces. And the nonlinear governing equations of motion were derived by using Lagrange equation. The displacements in three directions were represented by the characteristic orthogonal polynomial series and trigonometric functions. The effects of supporting stiffness and supported clearance on dynamic behavior of pipe wall were discussed. The results show that the existence of supported clearance may lead to the changing of stiffness of the pipe vibration system and the dynamic behaviors of the pipe system show nonlinearity and become more complex; for example, the amplitudefrequency curve of the foundation frequency showed hard nonlinear phenomenon. The chaos and bifurcation may emerge at some region of the values of stiffness and clearance, which means that the responses of the pressure thin-wall pipe segment would be more complex, including periodic motion, times periodic motion, and quasiperiodic or chaotic motions.
\end{abstract}

\section{Instruction}

Pressure pipe is commonly used as structural member or mechanical component in many engineering applications and chemical equipment, such as centrifugal compressor. For the inlet/outlet pipe of a centrifugal compressor, the pipe wall is commonly subjected to pulsed gas excitations and its restraint condition is always complex, which might cause nonlinear vibration of pipe. Because there is high pressure gas flowing through the pipe, strong vibration might cause pipe wall to break and it is a threat for air tightness and safety. It might lead to chemical gas leak and even an explosion. So the study of dynamic behavior of pressure pipe segment has an important signification for designing and maintenance of pipe system.

In actual work, the restraint condition of compressor pipe would be very complex. Firstly, installation error exists inevitably. Secondly, the long-playing vibration of the pipe system may lead to the deformation of pipe clamp and cause supported clearance between pipe wall and pipe clamp. The complex boundary condition would have effect on the dynamic characteristic of pipe. Up to now, researches on the nonlinear dynamic behaviors of pipe with nonlinear support were always based on pipe beam model. Beam model is often applied to the pipe which has large ratio of length to radius and large ratio of thickness to radius. And only the lateral movement of the pipe was studied by considering small circumferential mode. However, for the inlet/outlet pressure pipe of a centrifugal compressor, the ratio of length to radius and the ratio of thickness to radius are relatively small. They are always thin-wall pipes and their vibration is more complex. It would be more accurate to choose shell model to study the dynamic behaviors of pipe vibration system.

For shell model, many researches have been done in the literature review. In most of the literatures, boundary condition of shell is a much-discussed topic. Interrelated researches began from those about shells with classical boundaries (simply supported (S), clamped (C), free (F), and so on). And many methods were used to study free vibration and forced vibration of cylindrical shells based on different theories. Lee and Kwak [1] constructed a dynamic model for the free vibration analysis of a circular cylindrical 
shell by using the Rayleigh-Ritz method and compared the results based on different theories such as DonnellMushtari theory, Sanders theory, Flügge theory, Vlasov theory, Love-Timoshenko theory, and Reissner theory and the results under different boundary conditions. Lam and Loy [2] studied the effects of boundary conditions on the free vibration characteristic for a multilayered cylindrical shell based on Love's first approximation theory using Ritz method. The displacements were represented as combination beam functions with trigonometric functions. And nine kinds of classical boundaries were discussed. Based on threedimensional theory of elasticity, the boundary conditions of thick cylindrical shell, S-S and C-C, were studied by Loy and Lam $[3,4]$ using an energy minimization principle. And extensive frequency parameters were presented for a wide range of thickness to radius and thickness to length ratios. An improved version of the differential quadrature method was applied to solutions of cylindrical shell problems and the frequencies and fundamental frequencies for S-S, C-C, and C-S boundary conditions were determined [5]. Wave propagation method was also used in the vibration analysis of cylindrical shells by Zhang et al. [6]. This method is a noniterative method and the axial wave number of standing wave is determined approximately by the wave number of an equivalent beam with similar boundary conditions as the shell. Harr wavelet method was used by Xie et al. [7] for free vibrations of thin cylindrical shells with various boundary conditions based on Goldenveizer-Novozhilov shell theory and the displacements were represented by Haar wavelet series and their integrals in the axial direction and the Fourier series in the circumferential direction. Using the Flügge shell theory, Xie [8] employed transfer matrix method to analyze the natural characteristic of cylindrical shells and the precise integration technique was adopted in numerical calculation. Ebrahimi and Najafizadeh [9] used the methods of generalized differential quadrature (GDQ) and generalized integral quadrature (GIQ) to study the free vibration of a twodimensional functionally graded circular cylindrical shell based on Love's first approximation classical shell theory. Sun et al. [10] used Fourier series expansion method, which is derived by Stokes, transformation, to analyze the vibration characteristics of thin rotating cylindrical shells with several classical boundary conditions. Lopatin and Morozov [11] presented an analytical formula to calculate the fundamental frequencies for the laminated composite cylindrical shell with clamped edges based on the Fourier decomposition method. Wang et al. [12] studied the vibration of a cantilever cylindrical shell suffered a moving harmonic excitation and the method of averaging was used to analyze the nonlinear traveling wave responses. Dey and Ramachandra [13] presented the prebuckling analysis of a SS-SS composite cylindrical shell under periodic partial edge loadings by employing the Galerkin and Bolotin methods based on Donnell's nonlinear shallow shell theory and studied the influence of dynamic load amplitude on the nonlinear response.

The main work of compressor pipe is conveying gas, which causes high pressure on pipe wall. So the lateral gas pressure is an important loading condition for compressor pipe and can also impact the dynamic behavior of pipe.
Pressure shell was also a popular object for researchers. Isvandzibaei et al. studied natural frequency characteristics of a thin-walled multiple layered cylindrical shell under lateral pressure with symmetric [14] and asymmetric [15] boundary conditions using energy method. And the effects of geometrical parameter and pressure on natural frequencies were investigated. They [16] also employed this method into FGM cylindrical shell with rings support under symmetric uniform interior pressure distribution and ten different boundary conditions were discussed to study the natural characteristics. Based on Donnell-type classical shell theory, Forouzesh and Jafari [17] used The Hamilton's principle, differential quadrature, and Newmark method to solve the radial vibration problem of simply supported pseudoelastic shape memory alloy cylindrical shells under time-dependant internal pressure.

However, classical boundary could not represent general boundary conditions. Researchers shifted their attention to elastic boundary conditions. Massless springs were introduced to represent the interaction between the pipe end and the frame. And at each point the restraint condition is represented as four sets of independent springs, including three sets of linear springs and one set of rotational springs, and different boundary conditions can be obtained by setting different spring stiffness. Jin et al. [18-20] developed a series of methods to investigate free vibration of different cylindrical shells with general elastically restrained boundaries, such as composite laminated cylindrical shells and a three-layered passive constrained layer damping (PCLD) cylindrical shell, considering elastic restraints and intermediate ring supports. Qu et al. [21] applied the method of domain decomposition to the analysis of cylindrical shells. Free vibration of the stepped shells under different combinations of free, simply, clamp, and elastic support boundaries was discussed and force vibration response of stepped cylindrical shells was also studied with the influence of structural damping, stepped thickness, and boundary conditions. Based on Flügge's theory, Dai et al. [22] provided some numerical examples for the vibration analysis of circular cylindrical shells with various boundary conditions using the elastic equations. Chen et al. [23] investigated the vibration of cylindrical shell with nonuniform elastic boundary constraints using improved Fourier series method. Varying stiffness of boundary springs, point supported and partially supported boundary conditions were studied. Zhou et al. [24] analyzed free vibration of cylindrical shells with elastic boundary conditions by using the method of wave propagations based on Flügge classical thin shell theory. Shi et al. [25] used a spectro-geometric-Ritz method to study free vibration of open and closed shells with arbitrary boundary conditions. And the displacement components were also represented as a standard Fourier cosine series and several auxiliary functions. Sun and Liu [26, 27] employed the Rayleigh-Ritz method to study the effect of the variations of restraint stiffness on the natural characteristics of rotating cylindrical shells with and without ring-support based on the Sanders' shell theory and the displacements were considered as the characteristic orthogonal polynomial series.

Winkler and Pasternak foundation is also a topic researchers care about. Bakhtiari-Nejad and Mousavi Bideleh 


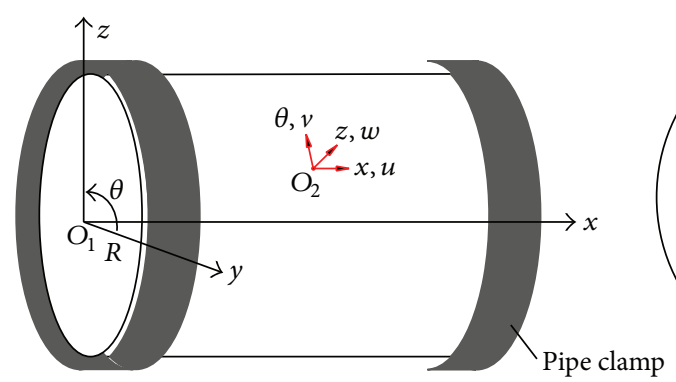

(a)

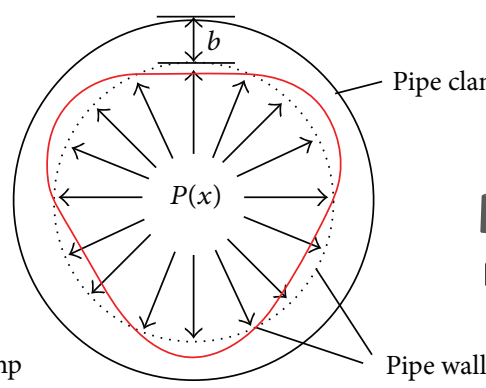

(b)

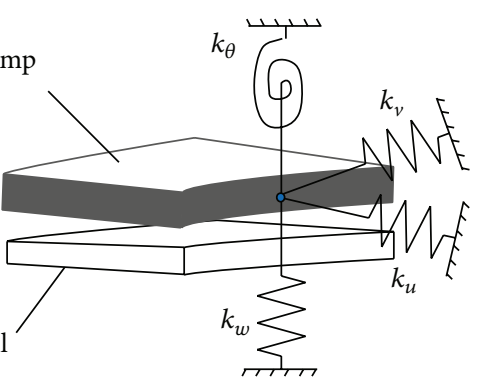

(c)

FIGURE 1: Simplified system dynamic model of pressure pipe with supported clearance at both ends.

[28] investigated nonlinear free vibration analysis of prestressed circular cylindrical shells placed on Winkler and Pasternak foundation and the effects of prestressed condition and elastic foundation on natural frequencies under various boundary conditions were analyzed. The RayleighRitz procedure and Perturbation methods are used to study amplitude-frequency characteristics of prestressed circular cylindrical shells. Shah et al. [29] employed wave propagation method and the approximate eigenvalues of characteristic beam functions to study the vibrations of functionally graded cylindrical shells based on the Winkler and Pasternak foundations.

As far as the study of circular cylindrical shell was considered, most of the existing works were limited to linear boundary conditions. However, nonlinear boundary conditions, such as those with supported clearance, could be encountered in many engineering applications. In present work, a short thin-wall cylindrical shell model with supported clearance at both ends was established for the pressure pipe of centrifugal compressor. Based on Sanders theory, Lagrange's equations had been written for the nonlinear vibration differential equations. In the analytical formulation, the Rayleigh-Ritz method with a set of displacement shape functions was used to deduce mass, damping, stiffness, and force matrices of the pipe system. The displacements in three directions were represented by the characteristic orthogonal polynomial series and trigonometric functions. By numerical calculation, dimensional spectrum, bifurcation diagram, time domain response graph, frequency spectrum plot, $s-v$ phase diagram, and Poincaré section with different parameters were obtained to analyze the effect of pulsed frequency, supporting stiffness, and supported clearance on dynamic characteristic of pressure pipe.

\section{Theory Formulation}

2.1. Simplified Dynamic Model of Pressure Thin-Wall Pipe Segment System. In actual work, due to poor installation quality or long time vibrating of the export pipe segment used in compressor, the pipe clamp may have a deformation or have the phenomenon of expansion, which can result in the supported clearance between pipe wall and pipe clamp, and it does harm to the pipe system. Considering the structure characteristic of export pipe of compressor that the ratio of length to diameter is little and the ratio of diameter to thickness is large, a pipe segment can be simplified as a cylindrical shell model shown in Figure 1. According to Sanders shell theory, the movement of each point on the pipe can be expressed by the movement of the points on the middle surface of pipe. So the cylindrical coordinate is established as illustrated in Figure 1(a), where $x, \theta$, and $z$ represent the axial direction, circumferential direction, and the radial direction, respectively. And the deformation of the cylindrical pipe segment with references to this coordinate system are denoted by $u, v$, and $w$ in $x, \theta$, and $z$ directions. And the pipe wall suffered uniform lateral gas pressure $P(x)$; the mode of the pressure is in Figure 1(b). In addition, the thickness, the length, and the mean radius of the cylinder are denoted by $h, L$, and $R$, respectively. The material of the pipe is assumed to be isotropic with the mass density $\rho$, Poisson's ratio $\mu$, and Young's modulus $E$.

Massless springs were introduced to describe the constraint condition of pipe wall from pipe clamp, and the constraint conditions at a point are represented as four sets of independent springs placed at the ends, including three sets of linear springs $\left(k_{u}, k_{v}\right.$, and $\left.k_{w}\right)$ and one set of rotational spring $\left(k_{\theta}\right)$ and they are shown in Figure 1(c) and the contact stiffness between pipe wall and pipe clamp is expressed by spring stiffness. It should be noted that the springs act in a uniform distributed manner at the both ends of the shell, and different boundary conditions can be obtained by setting different spring stiffness.

As shown in Figure 1(b), if the displacement of pipe vibration in radial direction was larger than the value of supported clearance denoted by $b$, the pipe end would be contacted with the pipe clamp and the supporting stiffness was assumed as $k$, and if the displacement of pipe vibration was less than the value $b$, the pipe clamp would be separated from the pipe wall and the supporting stiffness was zero. Therefore, the equivalent stiffness of constraint condition of pressure pipe with supported clearance at both ends could be assumed as

$$
\begin{aligned}
& k_{u}, k_{\theta}=0 \\
& k_{v}, k_{w}= \begin{cases}k, & w>b \\
0, & w \leq b\end{cases}
\end{aligned}
$$


So, the spring forces which represented the restraint forces are nonlinear forces and can be defined as

$$
\begin{aligned}
& f_{s u}=0, \\
& f_{s v}=\left.k_{v} v\right|_{\xi=0}+\left.k_{v} v\right|_{\xi=1}, \\
& f_{s \theta}=0, \\
& f_{s w}=k_{w}\left(\left.w\right|_{\xi=0}-b\right)+k_{w}\left(\left.w\right|_{\xi=1}-b\right),
\end{aligned}
$$

where $\xi$ is the dimensionless length and $\xi=x / L$ and $f_{s u}, f_{s v}, f_{s \theta}, f_{s w}$ are the spring forces in the directions of the corresponding springs. And the clearance model of the contact stiffness can be shown as in Figure 2.

2.2. Vibration Differential Equation of Pipe Segment. The kinetic energy for vibration of pressure pipe is given by

$$
\begin{aligned}
T & =\frac{\rho h L}{2} \\
& \cdot \int_{0}^{1} \int_{0}^{2 \pi}\left(\left(\frac{\partial u}{\partial t}\right)^{2}+\left(\frac{\partial v}{\partial t}\right)^{2}+\left(\frac{\partial w}{\partial t}\right)^{2}\right) R \mathrm{~d} \xi \mathrm{d} \theta .
\end{aligned}
$$

The strain energy $U_{\varepsilon}$ for the pipe based on Sanders' shell theory is

$$
\begin{aligned}
U_{\varepsilon} & =\int_{0}^{1} \int_{0}^{2 \pi}\left\{\frac{E h}{2\left(1-\mu^{2}\right)}\left[\left(\frac{1}{L} \frac{\partial u}{\partial \xi}\right)^{2}+\frac{2 \mu}{R L} \frac{\partial u}{\partial \xi}\left(\frac{\partial v}{\partial \theta}+w\right)+\frac{1}{R^{2}}\left(\frac{\partial v}{\partial \theta}+w\right)^{2}+\frac{1-\mu}{2}\left(\frac{1}{R} \frac{\partial u}{\partial \theta}+\frac{1}{L} \frac{\partial v}{\partial \xi}\right)^{2}\right]\right. \\
& \left.+\frac{E h^{3}}{24\left(1-\mu^{2}\right)}\left[\left(\frac{1}{L^{2}} \frac{\partial^{2} w}{\partial \xi^{2}}\right)^{2}+\frac{2 \mu}{R^{2} L^{2}} \frac{\partial^{2} w}{\partial \xi^{2}}\left(\frac{\partial^{2} w}{\partial \theta^{2}}-\frac{\partial v}{\partial \theta}\right)+\frac{1}{R^{4}}\left(\frac{\partial v}{\partial \theta}-\frac{\partial^{2} w}{\partial \theta^{2}}\right)^{2}+\frac{(1-\mu)}{2 R^{2}}\left(\frac{1}{2 R} \frac{\partial u}{\partial \theta}-\frac{3}{2 L} \frac{\partial v}{\partial \xi}+\frac{2}{L} \frac{\partial^{2} w}{\partial \xi \partial \theta}\right)^{2}\right]\right\}
\end{aligned}
$$

$\cdot R L \mathrm{~d} \xi \mathrm{d} \theta$.

And the pipe suffered uniform lateral gas pressure $P(x)$, which would cause prestress and prestrain of pipe wall. Assuming $P(x)=p$, the potential energy of the pipe can be derived

$$
U_{p}=-\int_{0}^{1} \int_{0}^{2 \pi} \frac{p}{2}\left[\left(\frac{\partial^{2} w}{\partial \theta^{2}}+w\right) w\right] L \mathrm{~d} \theta \mathrm{d} \xi
$$

In the course of work, the pipe wall may suffer the external loads on one point. Assume that the external loads of the unit area of pipe $f_{x}, f_{\theta}$, and $f_{z}$ in the directions of $u, v$, and $w$ can be denoted as

$$
\begin{aligned}
& f_{x}=0, \\
& f_{\theta}=0, \\
& f_{z}=F_{p} \delta\left(x-x_{0}\right) \delta\left(\theta-\theta_{0}\right) \sin (\omega t),
\end{aligned}
$$

where $F_{p}$ is the amplitude of the external load and is usually $2 \%$ to $5 \%$ of the value of the uniform distributed pressure and $\omega$ is the frequency of the external loads and it is also called pulse frequency of gas pressure.

The damping force can be denoted as

$$
\begin{aligned}
& f_{c x}=c_{e q} \dot{u}, \\
& f_{c \theta}=c_{e q} \dot{v}, \\
& f_{c z}=c_{e q} \dot{w} .
\end{aligned}
$$

In order to use the Rayleigh-Ritz method, the displacements $u, v$, and $w$ can be expressed in the terms of generalized coordinates:

$$
\begin{aligned}
u(\xi, \theta, t) & =\sum_{i=1}^{N T} a_{i}(t) \varphi_{i}^{u}(\xi) \cos (n \theta)=\mathbf{U}^{\mathrm{T}} \mathbf{q}_{U}, \\
v(\xi, \theta, t) & =\sum_{i=1}^{N T} b_{i}(t) \varphi_{i}^{v}(\xi) \sin (n \theta)=\mathbf{V}^{\mathrm{T}} \mathbf{q}_{V}, \\
w(\xi, \theta, t) & =\sum_{i=1}^{N T} c_{i}(t) \varphi_{i}^{w}(\xi) \cos (n \theta)=\mathbf{W}^{\mathrm{T}} \mathbf{q}_{W},
\end{aligned}
$$

where $\mathbf{q}_{U}, \mathbf{q}_{V}$, and $\mathbf{q}_{W}$ are the generalized coordinates or modal coordinates and $\mathbf{U}, \mathbf{V}$, and $\mathbf{W}$ are the displacement shape functions or the principal vibration modes which must satisfy the geometric boundary conditions. They are written by

$$
\begin{aligned}
& \mathbf{U}=\left[\varphi_{1}^{u}(\xi) \cos (n \theta), \varphi_{2}^{u}(\xi) \cos (n \theta), \ldots, \varphi_{N T}^{u}(\xi)\right. \\
& \cdot\cos (n \theta)]^{\mathrm{T}} ; \\
& \mathbf{q}_{U}= {\left[a_{1}(t), a_{2}(t), \ldots, a_{N T}(t)\right]^{\mathrm{T}} } \\
& \mathbf{V}=\left[\varphi_{1}^{v}(\xi) \sin (n \theta), \varphi_{2}^{v}(\xi) \sin (n \theta), \ldots, \varphi_{N T}^{v}(\xi)\right. \\
& \cdot\sin (n \theta)]^{\mathrm{T}} ; \\
& \mathbf{q}_{V}=\left[b_{1}(t), b_{2}(t), \ldots, b_{N T}(t)\right]^{\mathrm{T}} \\
& \mathbf{W}=\left[\varphi_{1}^{w}(\xi) \cos (n \theta), \varphi_{2}^{w}(\xi) \cos (n \theta), \ldots, \varphi_{N T}^{w}(\xi)\right.
\end{aligned}
$$




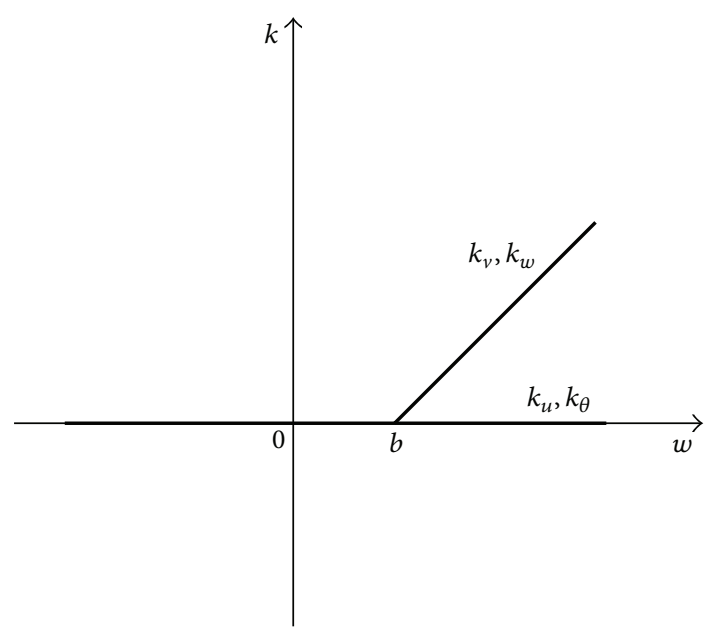

FIGURE 2: The clearance model of the spring forces.

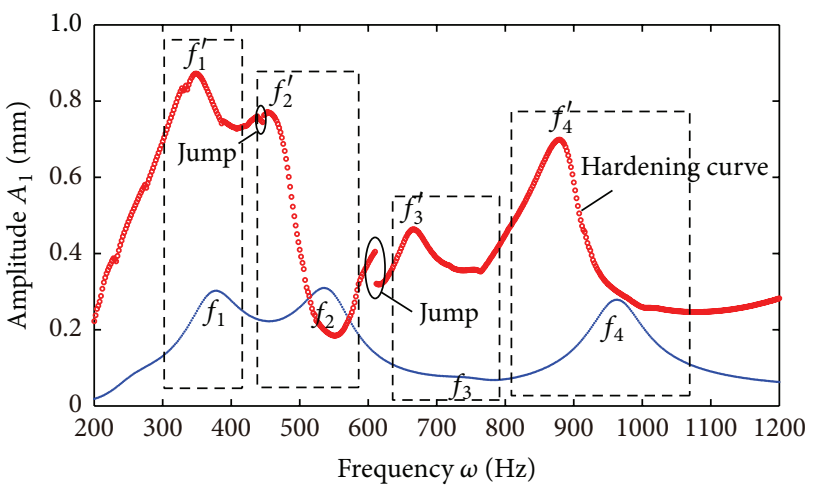

- Without supported clearance

- With supported clearance

FIGURE 3: A harmonic amplitude-frequency response curve.

$$
\begin{gathered}
\cdot \cos (n \theta)]^{\mathrm{T}} \\
\mathbf{q}_{W}=\left[c_{1}(t), c_{2}(t), \ldots, c_{N T}(t)\right]^{\mathrm{T}},
\end{gathered}
$$

where $a_{i}, b_{i}$, and $c_{i}$ are the coefficients and $\varphi_{i}^{u}(\xi), \varphi_{i}^{v}(\xi)$, and $\varphi_{i}^{w}(\xi)$ are characteristic orthogonal polynomials, which satisfy the geometric boundary conditions, generated by Gram-Schmidt process as shown in Appendix A. And NT is the number of terms for orthogonal polynomials truncated in practical calculation.

Then the kinetic energy, potential energy, and forces can be expressed in terms of the generalized coordinates and displacement shape functions. Substitute (7) into (3) to

$$
T=\frac{1}{2} \dot{\mathbf{q}}_{U}^{\mathrm{T}} \mathbf{M}_{1} \dot{\mathbf{q}}_{U}+\frac{1}{2} \dot{\mathbf{q}}_{V}^{\mathrm{T}} \mathbf{M}_{2} \dot{\mathbf{q}}_{V}+\frac{1}{2} \dot{\mathbf{q}}_{W}^{\mathrm{T}} \mathbf{M}_{3} \dot{\mathbf{q}}_{W},
$$

where $\mathbf{M}_{1}, \mathbf{M}_{2}$, and $\mathbf{M}_{3}$ are the modal mass matrices and they are listed in Appendix B.

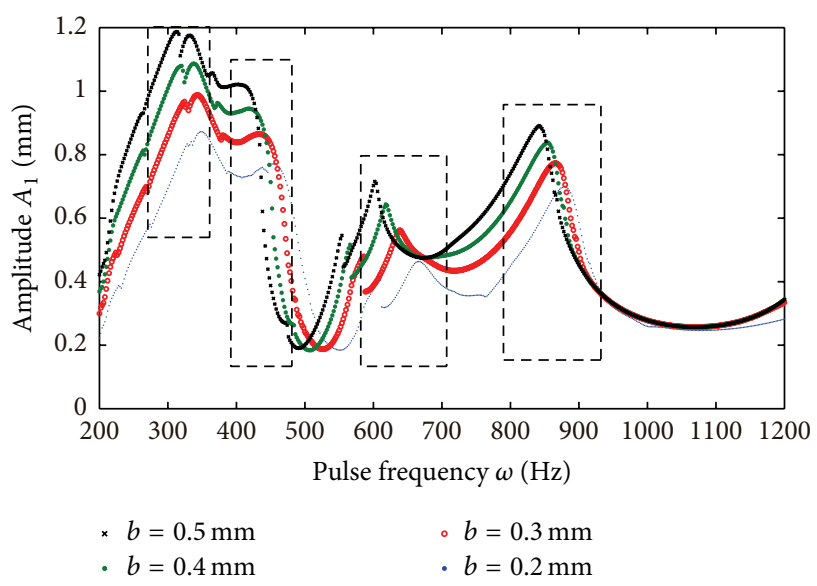

FIGURE 4: A harmonic amplitude-frequency response curve with different supported clearances.

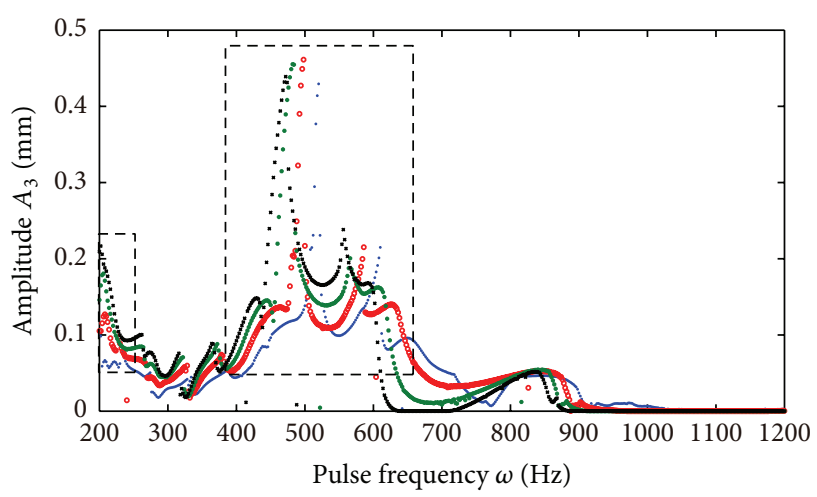

$$
\begin{array}{ll}
\times b=0.5 \mathrm{~mm} & \circ b=0.3 \mathrm{~mm} \\
\cdot b=0.4 \mathrm{~mm} & \cdot b=0.2 \mathrm{~mm}
\end{array}
$$

FIGURE 5: The third harmonic amplitude-frequency response curve with different supported clearances.

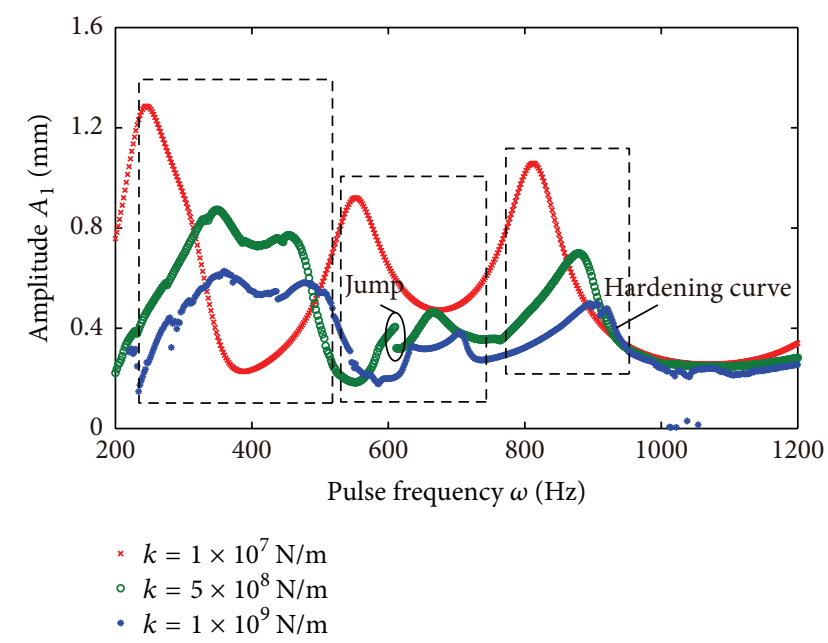

FIGURE 6: The first harmonic amplitude-frequency response curve with different supporting stiffness. 


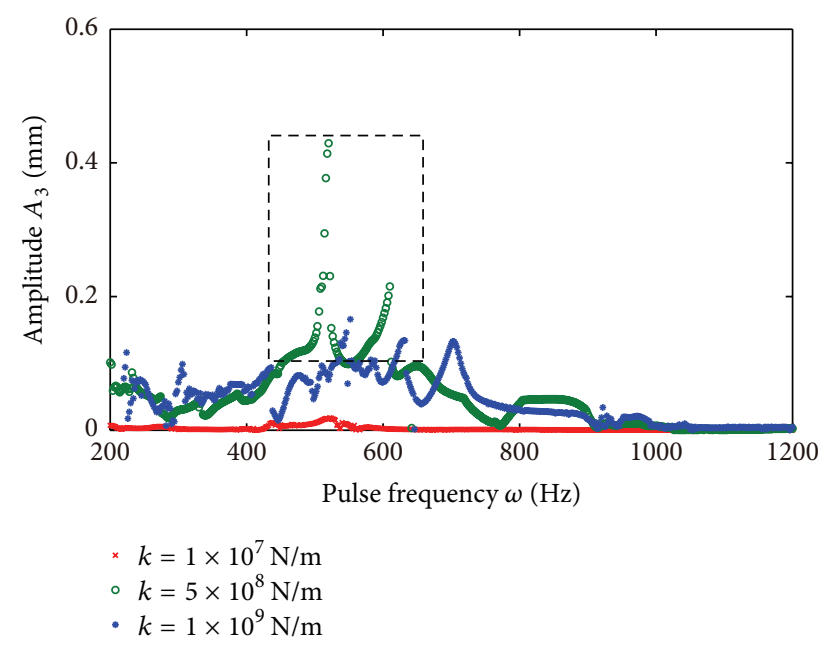

FIGURE 7: The third harmonic amplitude-frequency response curve with different supporting stiffness.

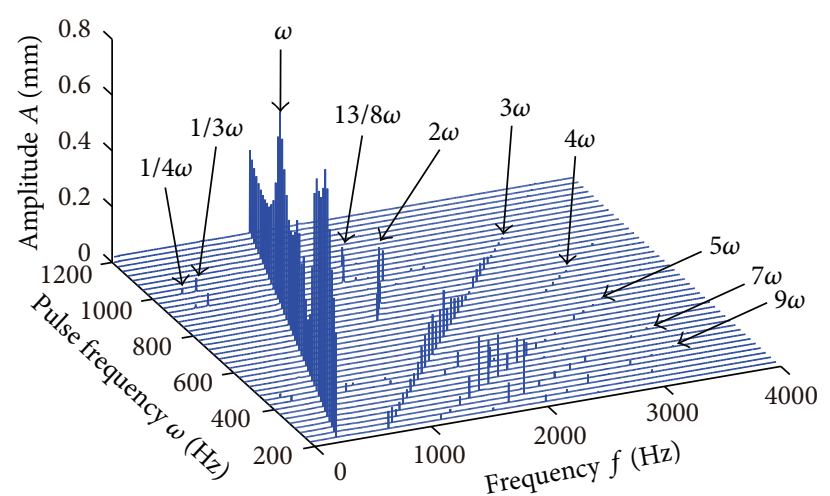

FIGURE 8: The three-dimensional spectrum diagram under the condition of $k=5 \times 10^{8} \mathrm{~N} / \mathrm{m}, b=0.2 \mathrm{~mm}$.

Substitute (8) into (4)-(5), respectively,

$$
\begin{aligned}
U_{\varepsilon}= & \frac{1}{2} \mathbf{q}_{U}^{\mathrm{T}} \mathbf{K}_{1} \mathbf{q}_{U}+\frac{1}{2} \mathbf{q}_{U}^{\mathrm{T}} \mathbf{K}_{2} \mathbf{q}_{V}+\frac{1}{2} \mathbf{q}_{U}^{\mathrm{T}} \mathbf{K}_{3} \mathbf{q}_{W} \\
& +\frac{1}{2} \mathbf{q}_{V}^{\mathrm{T}} \mathbf{K}_{4} \mathbf{q}_{V}+\frac{1}{2} \mathbf{q}_{V}^{\mathrm{T}} \mathbf{K}_{5} \mathbf{q}_{W}+\frac{1}{2} \mathbf{q}_{W}^{\mathrm{T}} \mathbf{K}_{6} \mathbf{q}_{W}, \\
U_{p}= & \frac{1}{2} \mathbf{q}_{W}^{\mathrm{T}} \mathbf{D} \mathbf{q}_{W},
\end{aligned}
$$

where $\mathbf{K}_{1}, \mathbf{K}_{2}, \mathbf{K}_{3}, \mathbf{K}_{4}, \mathbf{K}_{5}, \mathbf{K}_{6}$, and $\mathbf{D}$ are the modal stiffness matrices and they are also presented in Appendix B.

Substituting (8) into (6), the generalized exciting force can be got:

$$
\begin{aligned}
& \mathbf{F}_{f x}=0, \\
& \mathbf{F}_{f \theta}=0, \\
& \mathbf{F}_{f z} \\
& =\int_{0}^{1} \int_{0}^{2 \pi} \mathbf{W} F_{p} \sin (\omega t) \delta\left(\xi-\xi_{0}\right) \delta\left(\theta-\theta_{0}\right) L R \mathrm{~d} \theta \mathrm{d} \xi,
\end{aligned}
$$

where $\delta\left(\xi-\xi_{0}\right)$ is the Dirac function and one of its properties is

$$
\int_{a}^{b} f(x) \delta(x-c) d x=f(c), \quad a<c<b .
$$

Substituting (8) into (2), the generalized spring force can be written:

$$
\begin{aligned}
\mathbf{f}_{s u}\left(\mathbf{q}_{U}\right) & =0 \\
\mathbf{f}_{s v}\left(\mathbf{q}_{V}\right) & =\sum_{i=0}^{1} \int_{0}^{2 \pi} k_{v} \mathbf{V}_{\xi=i} \mathbf{V}_{\xi=i}^{\mathrm{T}} \mathbf{q}_{V} R \mathrm{~d} \theta, \\
\mathbf{f}_{s \theta}\left(\mathbf{q}_{W}\right) & =0 \\
\mathbf{f}_{s w}\left(\mathbf{q}_{W}\right) & =\sum_{i=0}^{1} \int_{0}^{2 \pi} k_{w} \mathbf{W}_{\xi=i}\left(\mathbf{W}_{\xi=i}^{\mathrm{T}} \mathbf{q}_{W}-b\right) R \mathrm{~d} \theta .
\end{aligned}
$$

Substituting (8) into (7), the generalized damping forces can be got:

$$
\begin{aligned}
& \left\{\mathbf{C}_{e q 1}, \mathbf{C}_{e q 2}, \mathbf{C}_{e q 3}\right\} \\
& \quad=\int_{A}\left\{c_{e q 1} \mathbf{U U}^{\mathrm{T}} \dot{\mathbf{q}}_{U}, c_{e q 2} \mathbf{V} \mathbf{V}^{\mathrm{T}} \dot{\mathbf{q}}_{V}, c_{e q 3} \mathbf{W} \mathbf{W}^{\mathrm{T}} \dot{\mathbf{q}}_{W}\right\} \mathrm{d} A .
\end{aligned}
$$

Lagrange equation with the Rayleigh-Ritz method will be used to determine the equation of motion of the pressure pipe. Lagrange equation is written by

$$
\frac{\mathrm{d}}{\mathrm{d} t}\left(\frac{\partial T}{\partial \dot{\mathbf{q}}}\right)-\frac{\partial T}{\partial \mathbf{q}}+\frac{\partial U}{\partial \mathbf{q}}=\mathbf{F}_{f}-\mathbf{F}_{c}-\mathbf{F}_{s},
$$

where $\mathbf{F}_{c}$ is the damping force vector, $\mathbf{F}_{s}$ is the spring force vector, and $\mathbf{F}_{f}$ is the external force vector:

$$
\begin{aligned}
& \mathbf{F}_{s}=\left[\begin{array}{lll}
\mathbf{f}_{s u}\left(\mathbf{q}_{U}\right) & \mathbf{f}_{s v}\left(\mathbf{q}_{V}\right) & \mathbf{f}_{s \theta}\left(\mathbf{q}_{W}\right)+\mathbf{f}_{s w}\left(\mathbf{q}_{W}\right)
\end{array}\right]^{\mathrm{T}}, \\
& \mathbf{F}_{f}=\left[\begin{array}{lll}
\mathbf{F}_{f x} & \mathbf{F}_{f \theta} & \mathbf{F}_{f z}
\end{array}\right]^{\mathrm{T}} .
\end{aligned}
$$

Substituting (8)-(15) into (16), the nonlinear vibration differential equation can be obtained as.

$$
\mathbf{M} \ddot{\mathbf{q}}+\mathbf{C} \dot{\mathbf{q}}+\mathbf{K q}+\mathbf{F}_{s}=\mathbf{F}_{f},
$$

where $\mathbf{M}, \mathbf{C}$, and $\mathbf{K}$ are the generalized mass matrix, the generalized damping matrix, and the generalized stiffness matrix. They can be written by

$$
\begin{aligned}
\mathbf{M} & =\left[\begin{array}{ccc}
\mathbf{M}_{1} & \mathbf{0} & \mathbf{0} \\
\mathbf{0} & \mathbf{M}_{2} & \mathbf{0} \\
\mathbf{0} & \mathbf{0} & \mathbf{M}_{3}
\end{array}\right], \\
\mathbf{C} & =\left[\begin{array}{ccc}
\mathbf{C}_{e q 1} & \mathbf{0} & \mathbf{0} \\
\mathbf{0} & \mathbf{C}_{e q 2} & \mathbf{0} \\
\mathbf{0} & \mathbf{0} & \mathbf{C}_{e q 3}
\end{array}\right],
\end{aligned}
$$




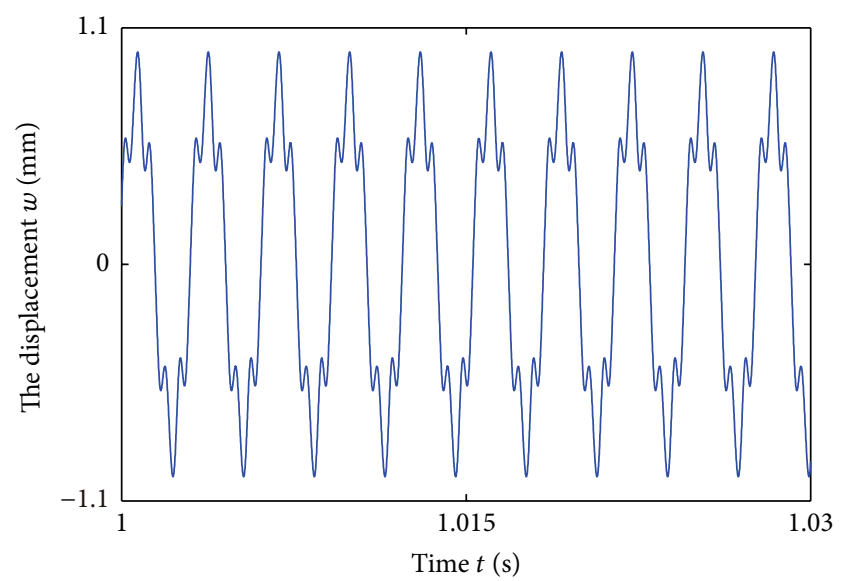

(a) Time domain response graph

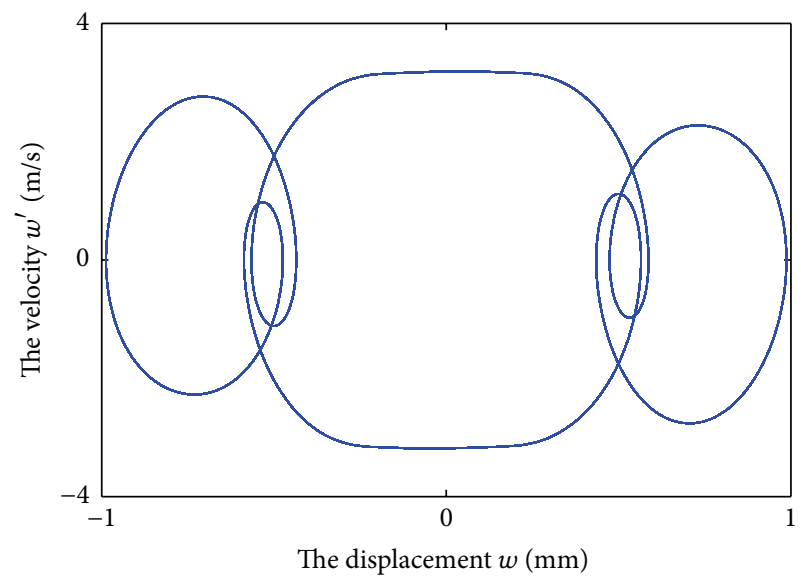

(c) $s-v$ phase diagram

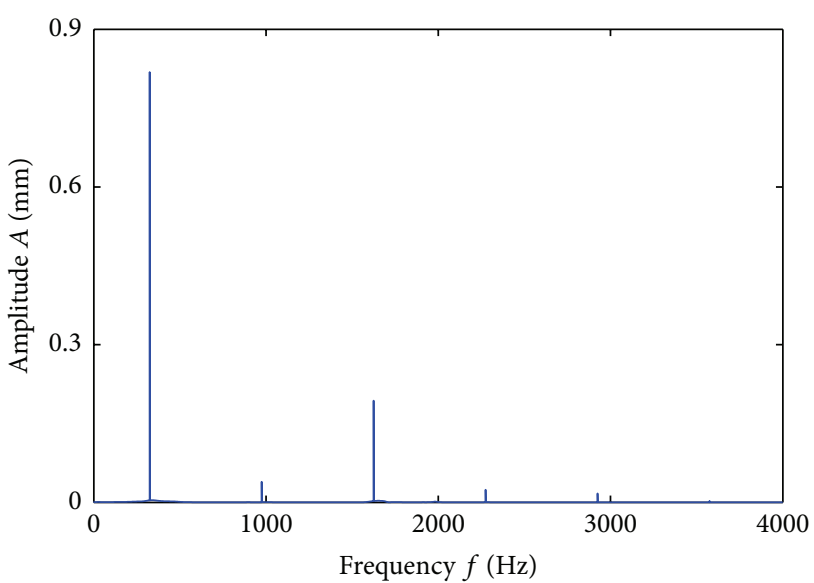

(b) Frequency spectrum plot

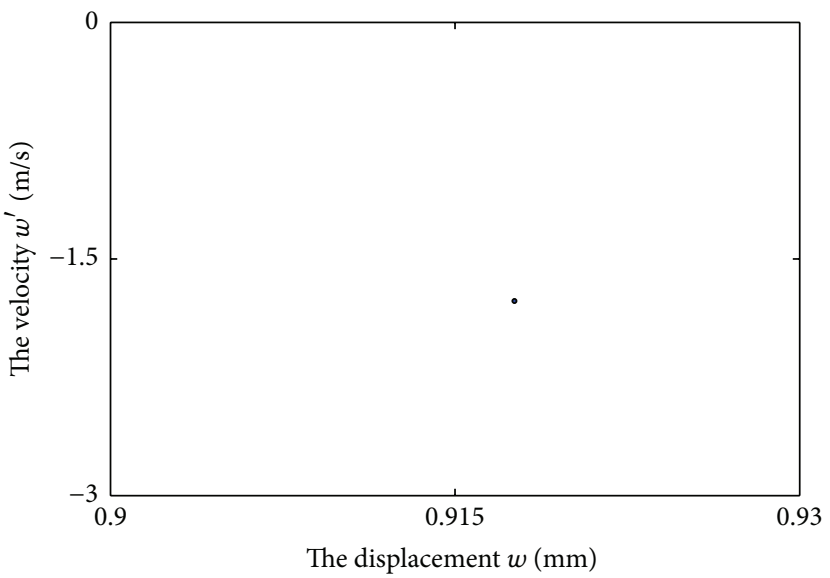

(d) Poincaré section

FIgURE 9: Kinetic characteristic curve under the condition $\omega=325 \mathrm{~Hz}$.

$$
\mathbf{K}=\left[\begin{array}{ccc}
\mathbf{K}_{1} & \frac{1}{2} \mathbf{K}_{2} & \frac{1}{2} \mathbf{K}_{3} \\
\frac{1}{2} \mathbf{K}_{2}^{\mathrm{T}} & \mathbf{K}_{4} & \frac{1}{2} \mathbf{K}_{5} \\
\frac{1}{2} \mathbf{K}_{3}^{\mathrm{T}} & \frac{1}{2} \mathbf{K}_{5}^{\mathrm{T}} & \mathbf{K}_{6}+\mathbf{H}
\end{array}\right] .
$$

Therefore, the model of pressure pipe segment with supported clearance at both ends is a nonlinear equation with piecewise linearity.

\section{Numerical Validation about Accuracy and Reliability}

To verify the accuracy and reliability of the present method, comparisons are made available in open literature through several numerical examples under the boundary condition without clearance, which are shown in Tables 1 and 2.

Firstly, what can be deduced is that the cylindrical shell is simply supported when $k$ is infinite. Good agreement can be seen with the results in [30], which was obtained by exact solutions based on Flügge's theory, and those in [22], which was solved by modified Fourier series method. The differences of the frequency parameters are very small. Secondly setting the spring stiffness to be zero, the cylindrical shell is free edges at two ends. The natural frequencies presented were compared with [22]. The differences of the frequency parameters between the two methods are very small. Therefore, the modal and method used in this paper have high accuracy and credibility, which provide the theoretical basis for the following calculation.

Usually, the restraint is not the classical boundary condition, so the case with elastic support was considered and the natural frequencies were listed in Table 3 , in which the magnitudes of the stiffness constant $(\widetilde{k})$ were assumed as 0,3 , $6,9,12$, and 15 , respectively.

\section{Amplitude-Frequency Characteristic Analysis of Pressure Pipe Segment with Supported Clearance}

To study the nonlinear dynamic behaviors of pressure pipe segment with supported clearance at both ends, amplitudefrequency responses of pipe wall vibration were analyzed and 


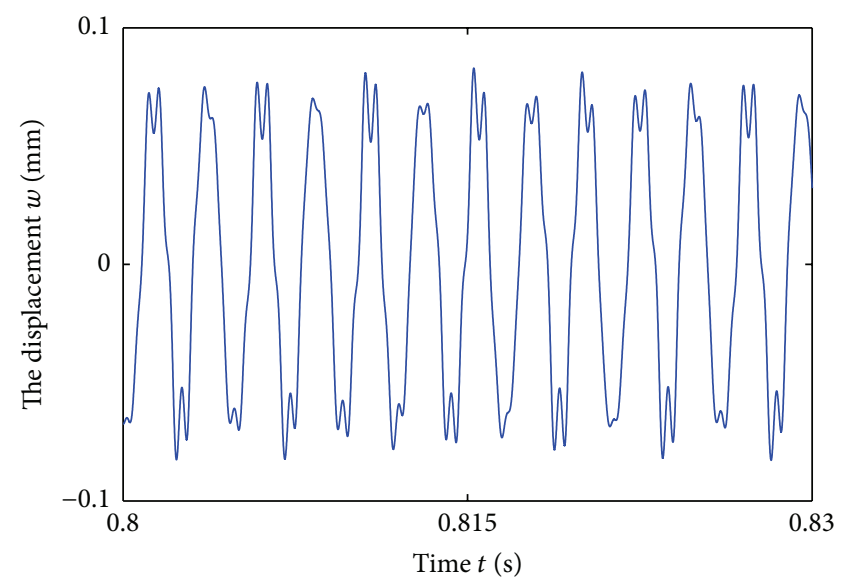

(a) Time domain response graph

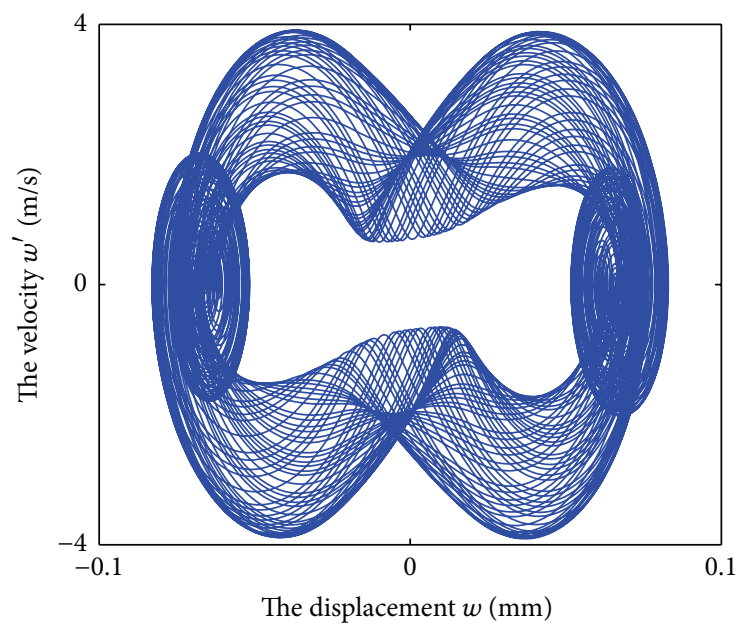

(c) $s-v$ phase diagram

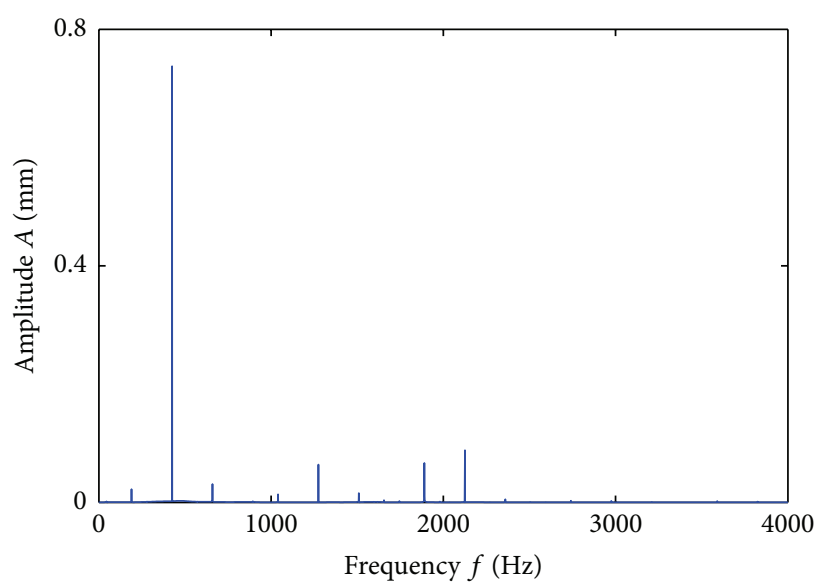

(b) Frequency spectrum plot

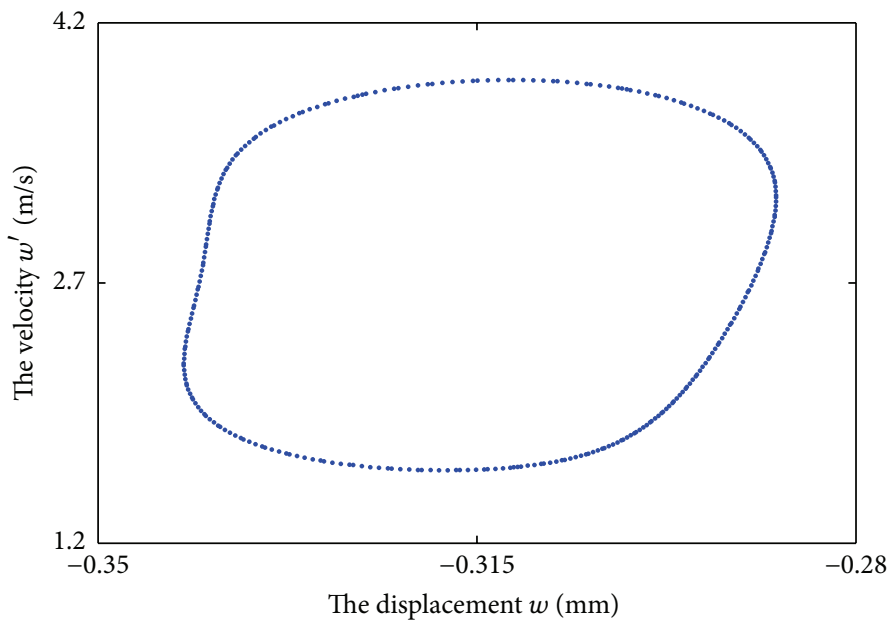

(d) Poincaré section

FIgURE 10: Kinetic characteristic curve under the condition $\omega=425 \mathrm{~Hz}$.

TABLE 1: Comparison of values of natural frequency parameter $\Omega=\omega R \sqrt{\rho\left(1-\mu^{2}\right) / E}$ for a circular cylindrical shell with simply supported boundary condition $(m=1, R / L=0.05, \mu=0.3)$.

\begin{tabular}{|c|c|c|c|c|}
\hline \multirow{2}{*}{$h / R$} & \multirow{2}{*}{$n$} & \multicolumn{3}{|c|}{$\Omega=\omega R \sqrt{\rho\left(1-\mu^{2}\right) / E}$} \\
\hline & & Present & Reference [30]/difference (\%) & Reference [22]/difference (\%) \\
\hline \multirow{4}{*}{0.05} & 1 & 0.0161028 & $0.0161065 / 0.02$ & $0.0161064 / 0.02$ \\
\hline & 2 & 0.0392714 & $0.0393038 / 0.08$ & $0.0393035 / 0.08$ \\
\hline & 3 & 0.1098115 & $0.1098527 / 0.04$ & $0.1098468 / 0.03$ \\
\hline & 4 & 0.2102772 & $0.2103446 / 0.03$ & $0.2103419 / 0.03$ \\
\hline \multirow{4}{*}{0.002} & 1 & 0.0161011 & $0.0161011 / 0.00$ & $0.0161023 / 0.01$ \\
\hline & 2 & 0.0054530 & $0.0054532 / 0.00$ & $0.0054547 / 0.03$ \\
\hline & 3 & 0.0050415 & $0.0050419 / 0.01$ & $0.0050427 / 0.02$ \\
\hline & 4 & 0.0085339 & $0.0085341 / 0.00$ & $0.0085344 / 0.01$ \\
\hline
\end{tabular}

the effects of supported clearance and supporting stiffness on amplitude-frequency characteristics were discussed in detail.

4.1. Nonlinearity of Pressure Pipe Segment with Supported Clearance at Both Ends. Figure 3 presents the curves of a harmonic amplitude-frequency response, where "o" means one case with a supported clearance at bout ends and "•" means the other case without supported clearance. As shown in Figure 3, due to existence of supported clearance at both ends, the response shows obvious strong nonlinearity. The supported clearance leads to a reduction of the stiffness of system and the early arrival of the resonance peaks, which is 
TABLE 2: Comparison of values of natural frequency parameter $\Omega=\omega R \sqrt{\rho\left(1-\mu^{2}\right) / E}$ for a circular cylindrical shell with free-free boundary condition $\left(L=0.502 \mathrm{~m}, R=0.0635 \mathrm{~m}, h=0.00163 \mathrm{~m}, \mu=0.28, E=2.1 e 11 \mathrm{~N} / \mathrm{m}^{2}\right.$, and $\left.\rho=7800 \mathrm{~kg} / \mathrm{m}^{3}\right)$.

\begin{tabular}{ccccccc}
\hline$n$ & & $m=1$ & & \multicolumn{2}{c}{$m=2$} \\
Reference [22] & Difference (\%) \\
\hline 1 & Present & Reference [22] & Difference (\%) & Present & 4830.6 & 0.01 \\
2 & 2479.2 & 2479.3 & 0.01 & 27830.2 & 278.58 & 0.38 \\
3 & 269.4 & 269.3 & 0.04 & 773.2 & 771.62 & 0.20 \\
4 & 761.9 & 761.0 & 0.12 & 1473.7 & 1469.3 & 0.30 \\
5 & 1460.9 & 1458.6 & 0.15 & 2376.3 & 2369.0 & 0.30 \\
\hline
\end{tabular}

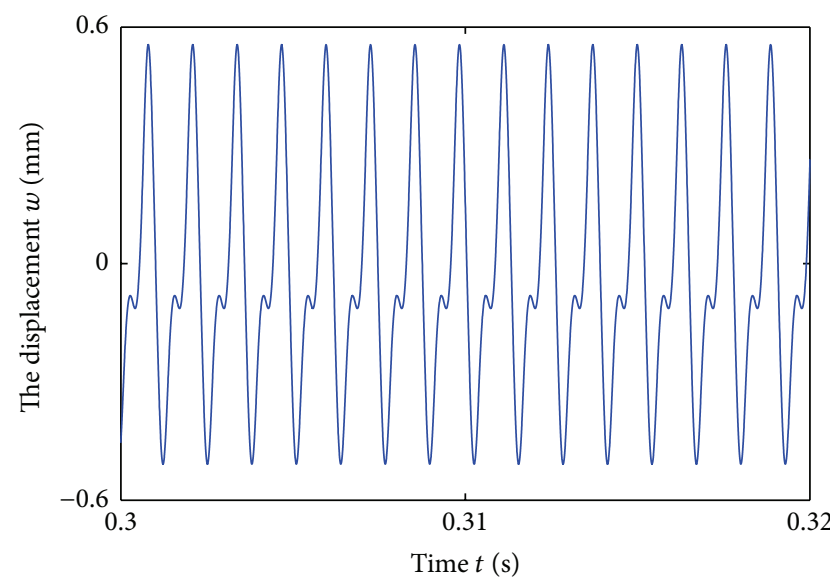

(a) Time domain response graph

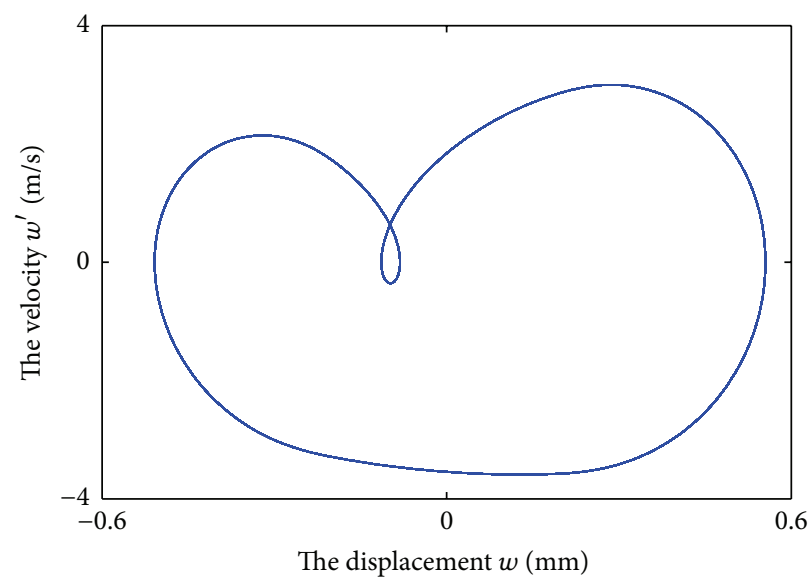

(c) $s-v$ phase diagram

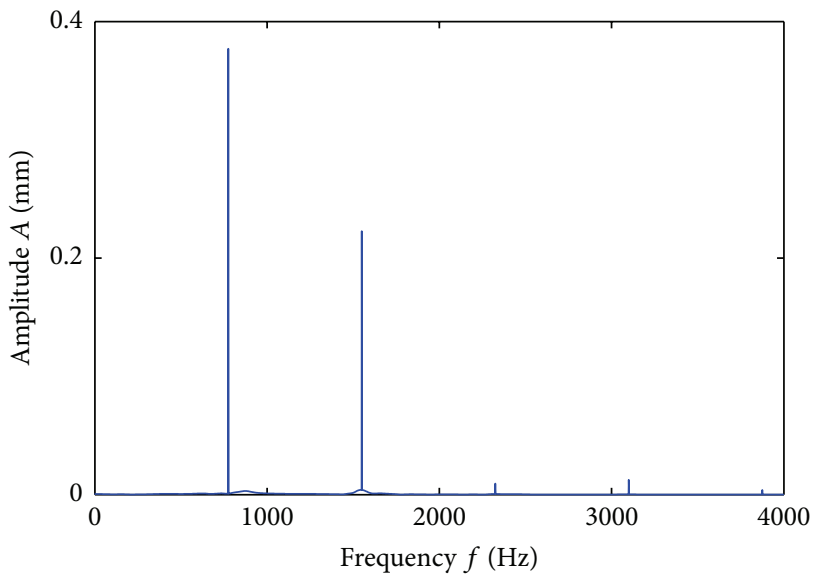

(b) Frequency spectrum plot

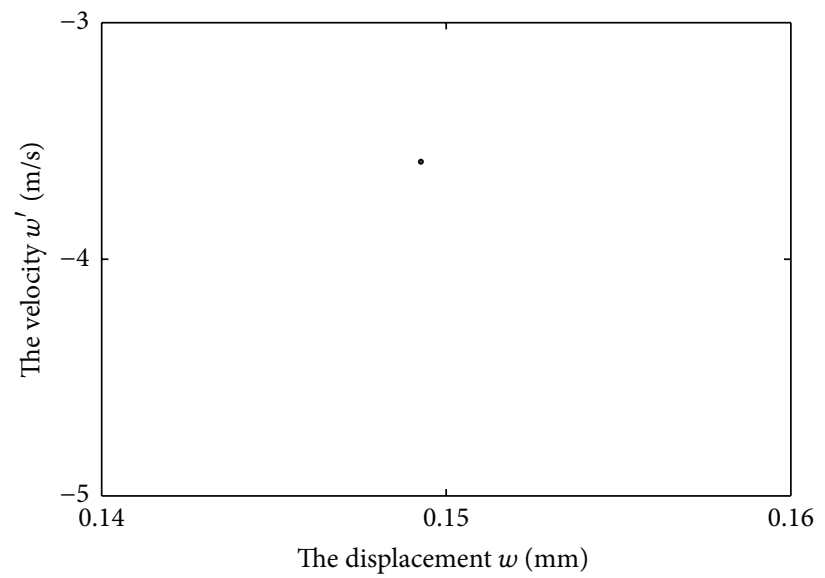

(d) Poincaré section

FIgURE 11: Kinetic characteristic curve under the condition $\omega=775 \mathrm{~Hz}$.

well presented in the dotted boxes. The amplitude-frequency curve skews to the right and it is called "hardening curve," and the amplitudes of the resonance peaks increase relatively. As the increasing of frequency, the phenomena of strong nonlinearity get more obvious. What is more, an obvious jump phenomenon emerges in the curve of amplitudefrequency response at the changing process of frequency. For example, the amplitude jumps up when $\omega$ reaches $446 \mathrm{~Hz}$ and jumps down when $\omega$ increases to $602 \mathrm{~Hz}$. Especially at $602 \mathrm{~Hz}$, the jumping phenomenon is more obvious, which reflects a great relative motion of pipe wall. The greater relative motion may have a great impact on the pipe wall and tend to produce great stress, cause fatigue failure, and reduce the working life of pipe. At another frequency $446 \mathrm{~Hz}$, the jumping phenomenon is not obvious and it indicates that the relative movement is not too large.

4.2. Effect of Supported Clearance on Amplitude-Frequency Characteristic of Pressure Pipe Segment. For pressure pipe segment, supported clearance is an important parameter which has a great influence on the system dynamic 


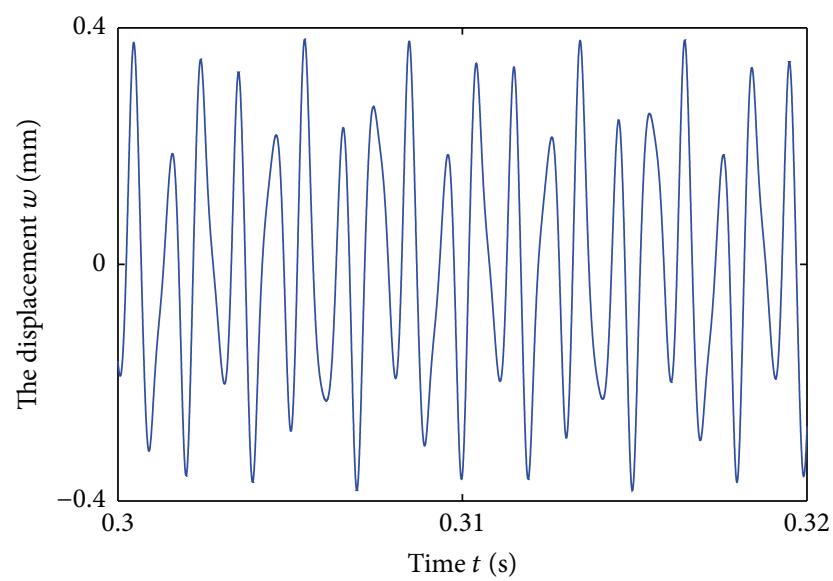

(a) Time domain response graph

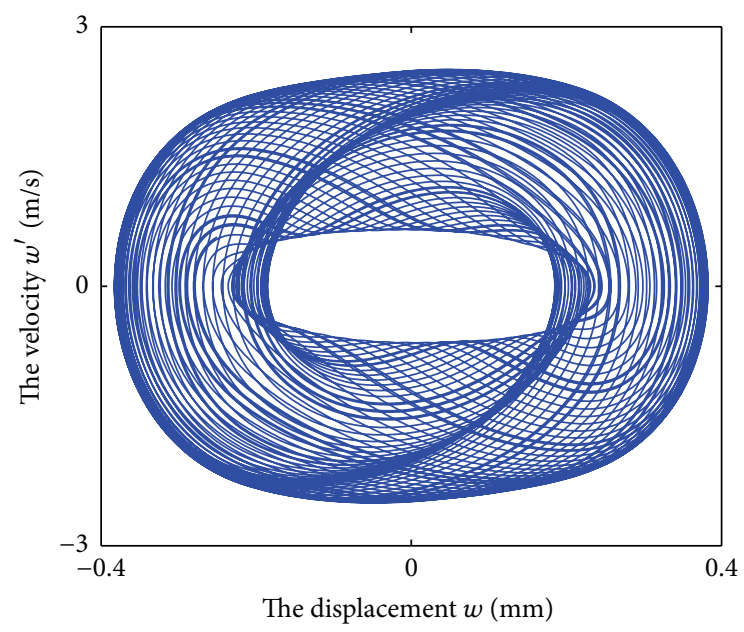

(c) $s-v$ phase diagram

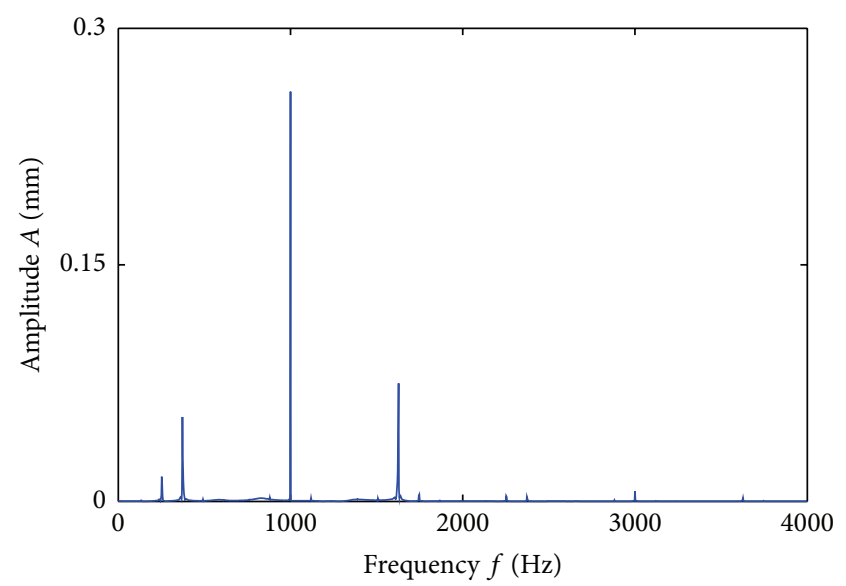

(b) Frequency spectrum plot

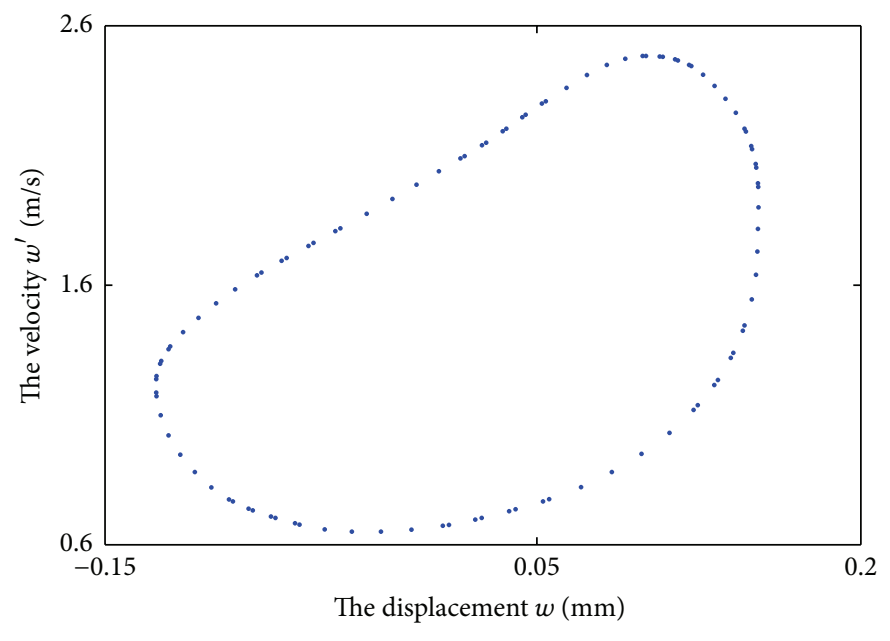

(d) Poincaré section

FIGURE 12: Kinetic characteristic curve under the condition $\omega=1000 \mathrm{~Hz}$.

TABle 3: Natural frequencies $(\mathrm{Hz})$ for an elastically supported cylindrical shell $(L=2 \mathrm{~m}, R=0.5 \mathrm{~m}, h=0.01 \mathrm{~m}, \mu=0.3$, $E=2.06 e 11 \mathrm{~N} / \mathrm{m}^{2}, \rho=7850 \mathrm{~kg} / \mathrm{m}^{3}$, and $\left.n=3\right)$.

\begin{tabular}{ccccccc}
\hline Mode & $\widetilde{k}=0$ & $\widetilde{k}=3$ & $\widetilde{k}=6$ & $\widetilde{k}=9$ & $\widetilde{k}=12$ & $\widetilde{k}=15$ \\
\hline$m=1$ & 228.18 & 228.19 & 228.89 & 247.94 & 249.44 & 249.44 \\
$m=2$ & 228.71 & 228.72 & 230.82 & 376.01 & 406.03 & 406.03 \\
$m=3$ & 318.05 & 318.05 & 319.91 & 557.74 & 642.86 & 642.86 \\
\hline
\end{tabular}

characteristics. This section gives the analysis of the amplitude-frequency characteristics of the pipe wall by changing the value of the supported clearance and keeping other parameters invariable. The curves in Figure 4 show a harmonic amplitude-frequency characteristics of the pipe wall at the point $(\xi=1, \theta=0)$. As demonstrated, the system displays the obvious nonlinear characteristics under every clearance. The amplitude-frequency curves tilt to right at the resonance frequency region, and the obvious jump phenomena occur in the continuously varying process of pulse frequency. It can be found that the support clearance has a great impact on the resonance region and resonance amplitude of the system, which can be seen in the dotted boxes. The greater supported clearance means smaller equivalent system stiffness, which causes larger amplitude of the resonance region and smaller excitation frequency needed to achieve the desired resonant. On the contrary, the smaller the supported clearance of the system is, the more obvious hardening phenomenon the amplitude-frequency curve shows. The amplitudes of the third harmonic vary with the pulse frequency under several different supported clearances which are shown in Figure 5. In a certain excitation frequency region, mainly in the low-frequency range, the amplitudes of third harmonic components of the system are very large. Due to the presence of higher harmonics, the response of the pipe segment would be more complex. Therefore, in the procedure of designing, installing, and routine maintenance of the equipment, the existence of the support clearance should be noticed.

\subsection{Effect of Supporting Stiffness on Amplitude-Frequency} Characteristic of Pressure Pipe Segment. For pressure pipe, the supporting stiffness is also an important parameter which affects the amplitude-frequency response characteristics of 


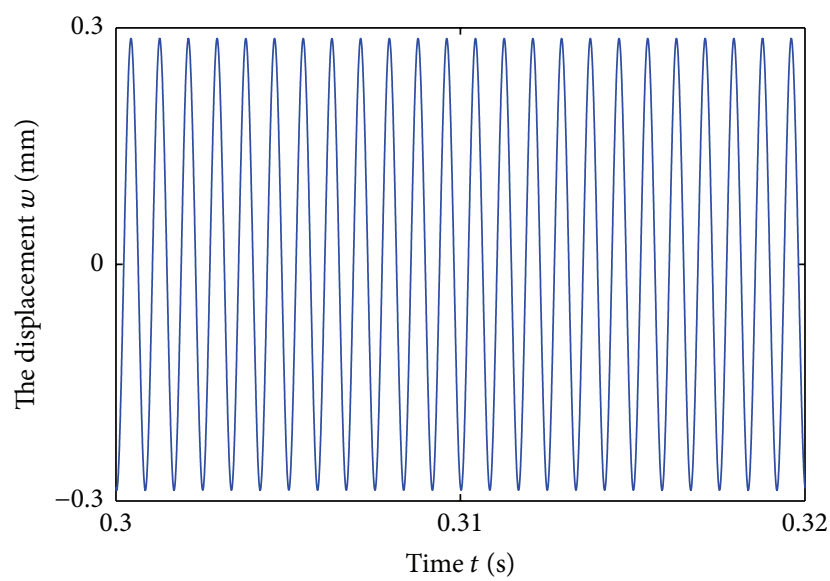

(a) Time domain response graph

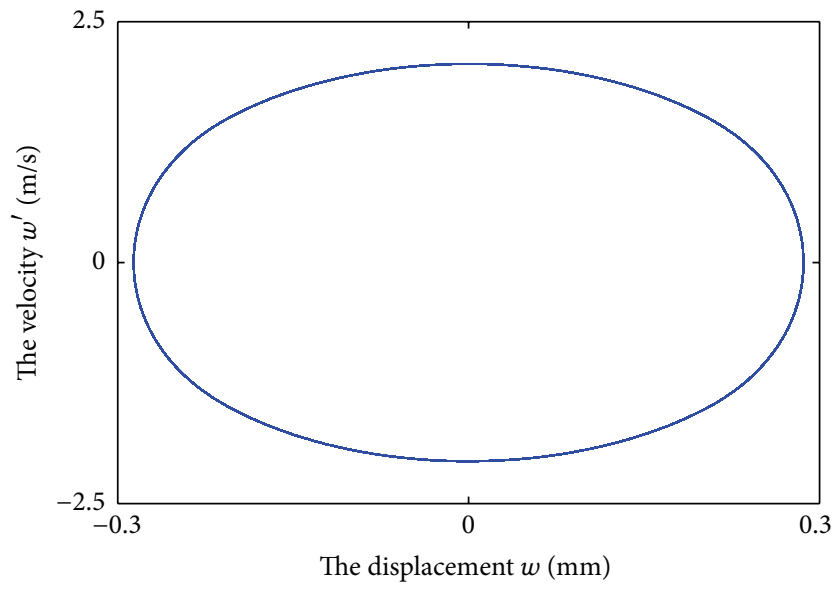

(c) $s-v$ phase diagram

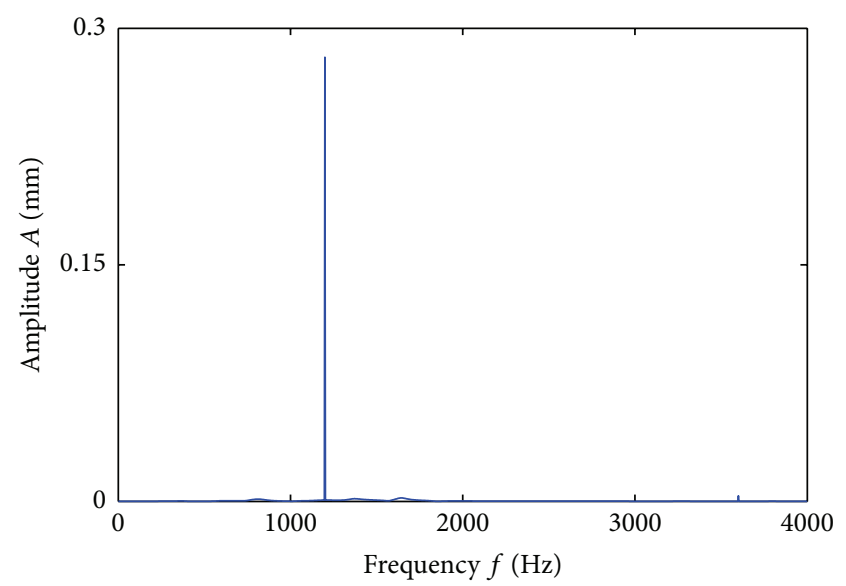

(b) Frequency spectrum plot

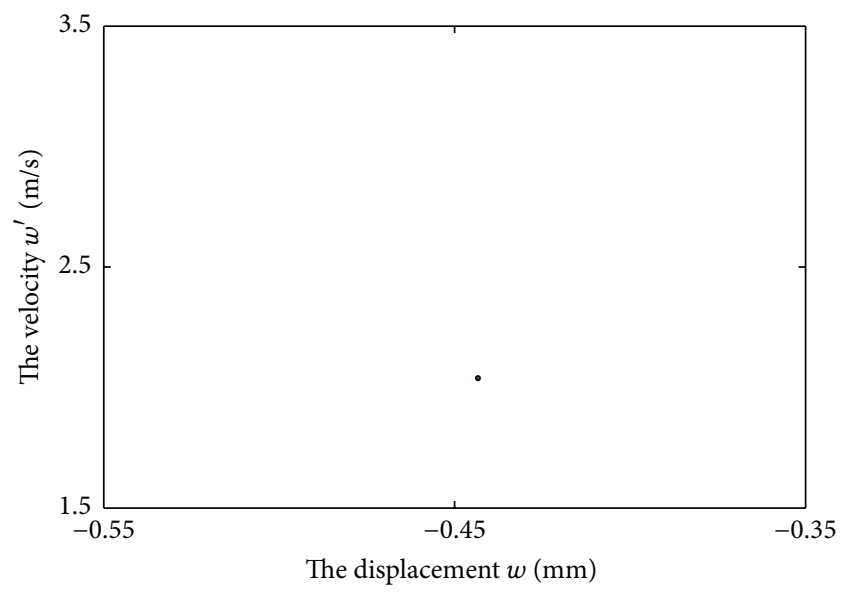

(d) Poincaré section

FIGURE 13: Kinetic characteristic curve under the condition $\omega=1200 \mathrm{~Hz}$.

pipe vibration system. In this part, the amplitude-frequency characteristics of the pipe wall were analyzed by only changing the system supporting stiffness and keeping other parameters invariable. Figure 6 displays the curves of the first harmonic amplitude of the response varying with the pulse frequency at the case of the clearance $b=0.2 \mathrm{~mm}$. As shown in the dotted boxes in Figure 6, the supporting stiffness has a great influence on the resonance frequency and the resonance amplitude, especially in the higher frequency region. Because of the existence of the supported clearance, the amplitudefrequency curve is tilted to the right, which shows a hard nonlinear phenomenon, and what should be noticed is that when the stiffness $k=5 \times 10^{8} \mathrm{~N} / \mathrm{m}$, the curve shows obvious jump phenomenon at the pulsation frequency $602 \mathrm{~Hz}$. When the supporting stiffness decreases, the resonance frequency reduces and the curve begins to slowly return back, which means that hard nonlinear phenomenon gradually weakened. But the response amplitude increases gradually. Figure 7 shows that the third harmonic amplitude varies with the supporting stiffness. And in a certain frequency region which is shown in the dotted box, the amplitudes of third harmonic components are very large for $k=1 \times 10^{7} \mathrm{~N} / \mathrm{m}$ and $k=$ $5 \times 10^{8} \mathrm{~N} / \mathrm{m}$, which make the response complex. So in the process of designing pipe clamp, the supporting stiffness also needs to be paid attention on.

\section{Dynamic Response Analysis of Pressure Pipe Segment with Supported Clearance}

5.1. Effect of Pulse Frequency on Dynamic Response of Pressure Pipe Segment. To fully analyze nonlinear dynamic behaviors of the pressure pipe vibration system with supported clearance at both ends, response analysis, parameter comparison, and other aspects were investigated. Through numerical simulating and calculating, the three-dimensional waterfall spectrogram was obtained. And time domain response graph, frequency spectrum plot, displacement-velocity phase diagram, and Poincaré section were also given to analyze the dynamic characteristics under different frequencies.

Figure 8 is the three-dimensional waterfall spectrogram in radial direction at the point $(\xi=1, \theta=0)$. As demonstrated, because of the existence of supported clearance, abundant constituents of frequency multiplication and frequency demultiplication appear in the vibration response of the pressure pipe, such as $\omega, 2 \omega, 3 \omega, 4 \omega, 5 \omega, 7 \omega, 1 / 2 \omega, 1 / 3 \omega$, and $13 / 8 \omega$. It can be found that $\omega, 3 \omega$, and $5 \omega$ are the obvious 


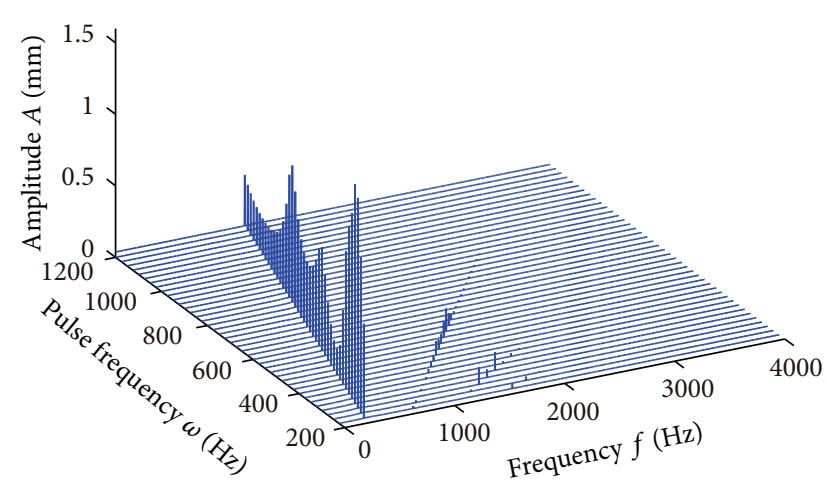

(a) $k=1 \times 10^{8} \mathrm{~N} / \mathrm{m}$

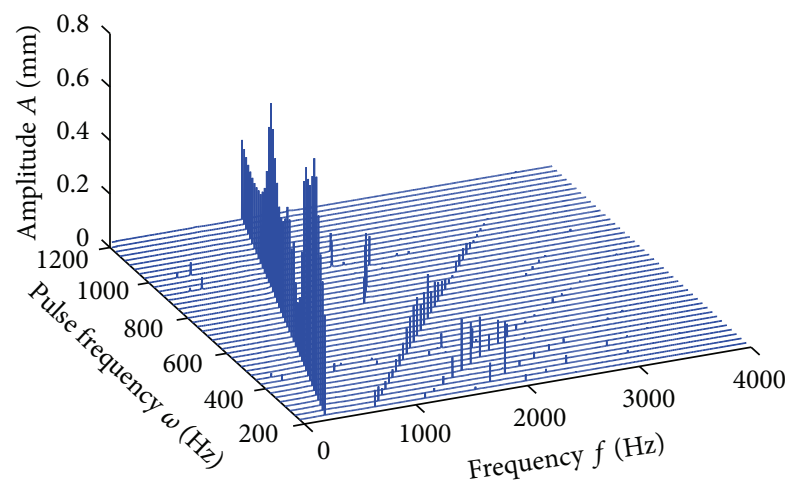

(b) $k=5 \times 10^{8} \mathrm{~N} / \mathrm{m}$

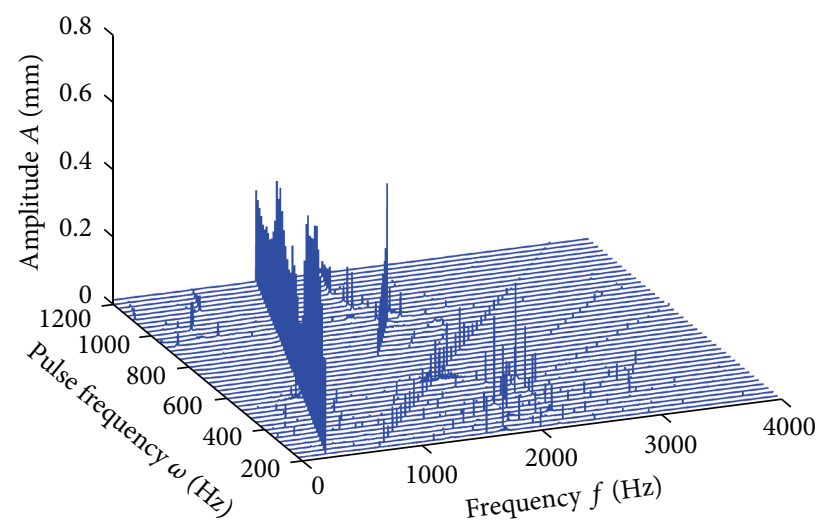

(c) $k=1 \times 10^{9} \mathrm{~N} / \mathrm{m}$

FIGURE 14: The dimensional spectrum under different stiffness.

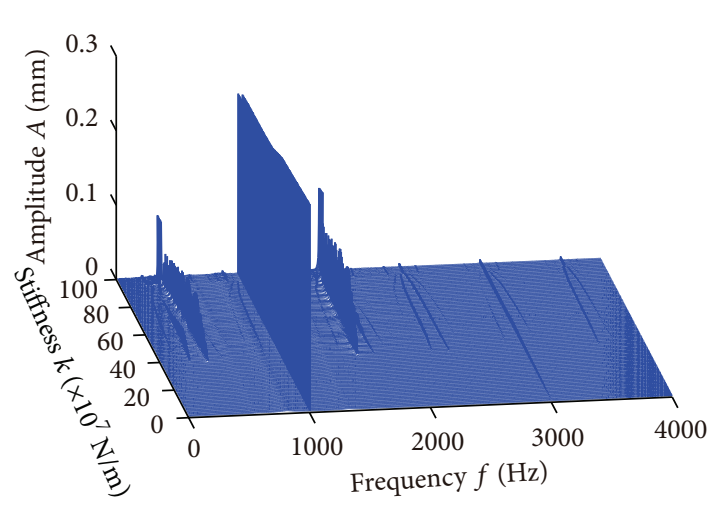

(a) The dimensional spectrum

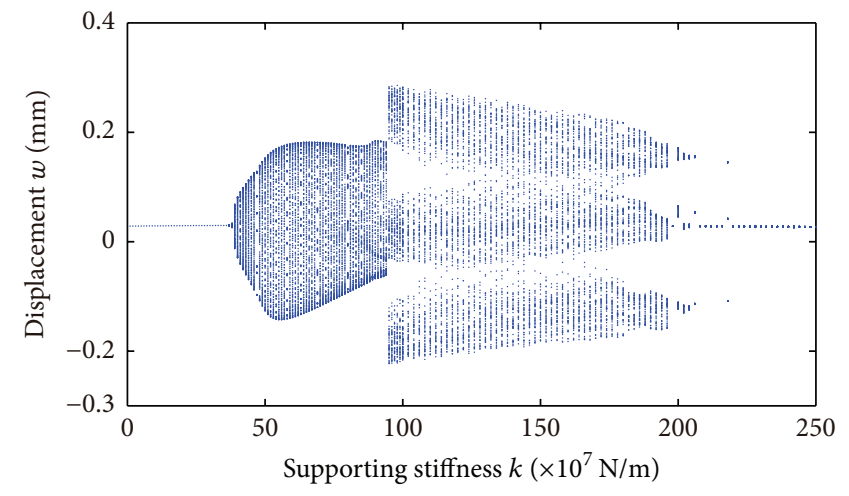

(b) The bifurcation diagram

Figure 15: The dimensional spectrum and the bifurcation diagram under different stiffness.

frequencies under arbitrary pulse excitation frequency. And the peak of amplitudes will appear in the spectrogram at the frequency $\omega$. When $200 \mathrm{~Hz} \leq \omega \leq 400 \mathrm{~Hz}$, the main frequencies are $\omega, 3 \omega, 5 \omega, 7 \omega$, and $9 \omega$ and every frequency does great contribution to vibration energy. In the process of changing frequency, the peak of amplitude appears at $\omega$, the amplitude of $3 \omega$ remains unchanged, that of $5 \omega$ and $7 \omega$ increase firstly and then decrease, and that of $9 \omega$ is so small that it could be neglected. When $400 \mathrm{~Hz} \leq \omega \leq 500 \mathrm{~Hz}$, the constituents of frequency demultiplication appear in the vibration system. The amplitude of $3 \omega$ is gradually increasing and those of $5 \omega$ and $7 \omega$ continue decreasing. When $500 \mathrm{~Hz}$ $\leq \omega \leq 725 \mathrm{~Hz}$, expect $\omega, 3 \omega$, and $5 \omega$, all the constituents of frequency doubling and frequency demultiplication vanish. The amplitude of $3 \omega$ begins to reduce after it gets a certain value and the amplitude of $5 \omega$ also gradually reduces and then vanishes. When $725 \mathrm{~Hz} \leq \omega \leq 900 \mathrm{~Hz}, 2 \omega$ and $4 \omega$ appear and $5 \omega$ vanishes. The energy of the pipe vibration system is mainly contributed to $1 \omega$ and $2 \omega$. When $900 \mathrm{~Hz} \leq \omega \leq 1000 \mathrm{~Hz}, 2 \omega$ and $4 \omega$ rapidly disappeared and there are new constituents 


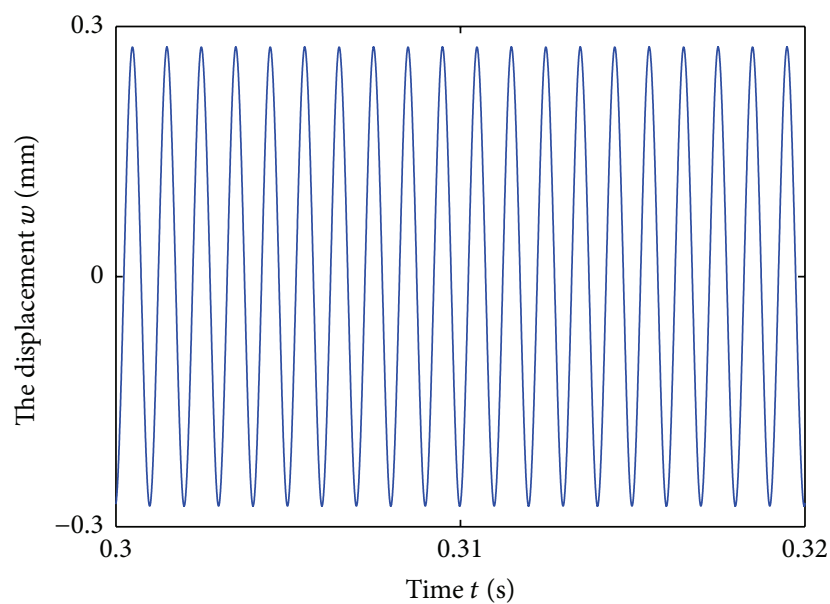

(a) Time domain response graph

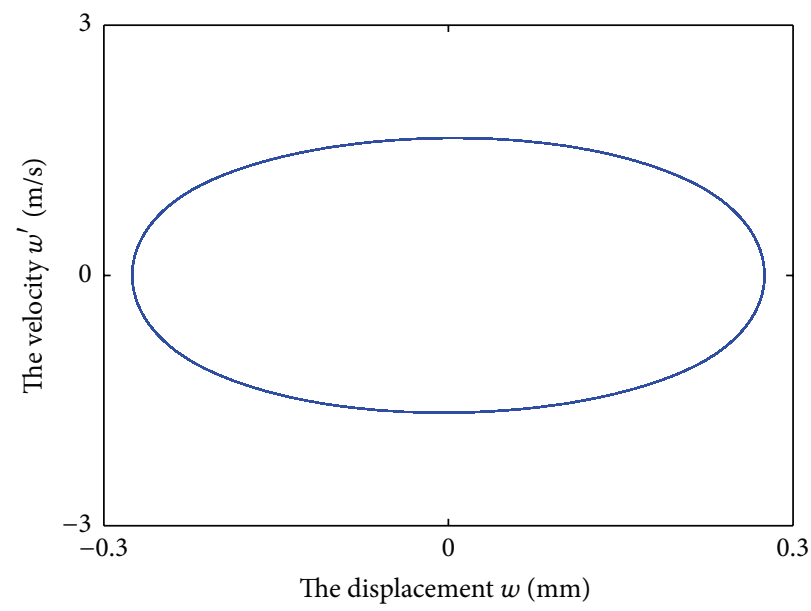

(c) $s-v$ phase diagram

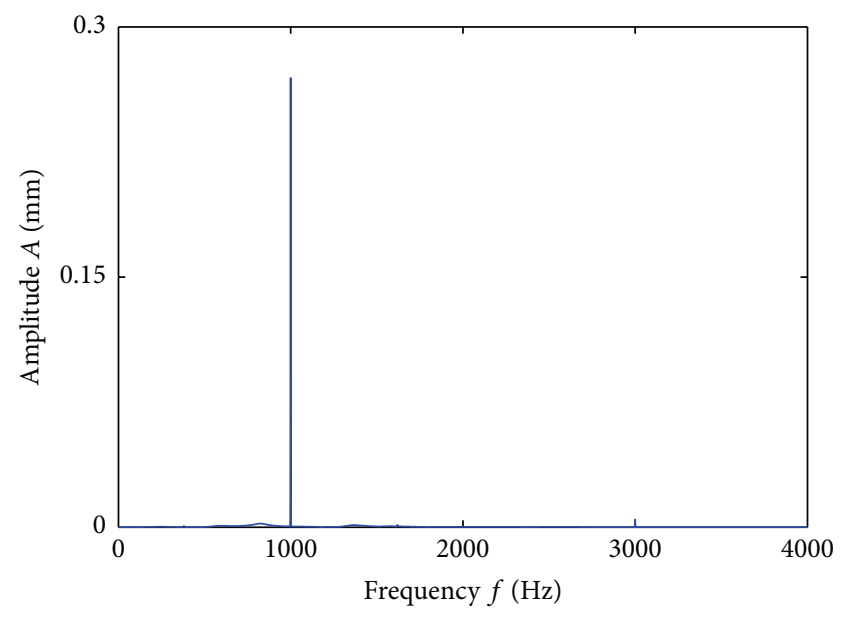

(b) Frequency spectrum plot

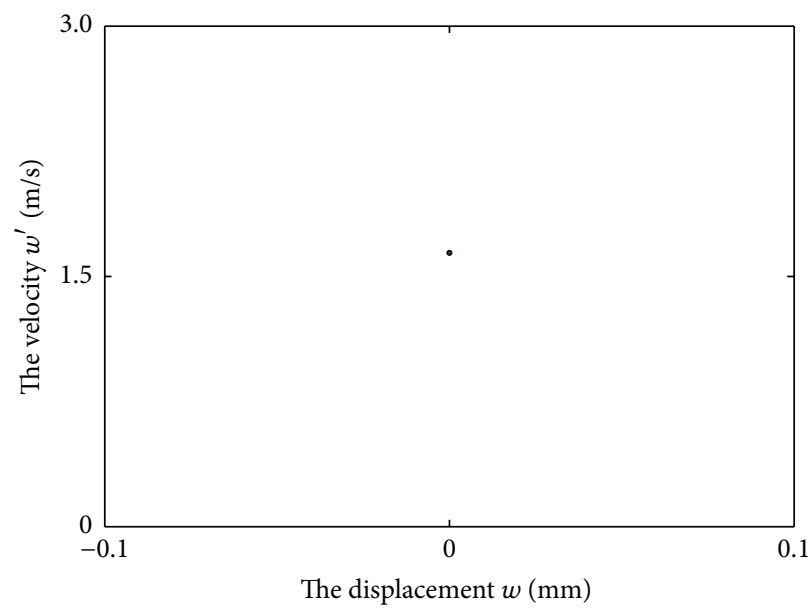

(d) Poincaré section

FIGURE 16: Kinetic characteristic curve under the condition $k=3 \times 10^{8} \mathrm{~N} / \mathrm{m}$.

that appeared such as $1 / 3 \omega, 1 / 4 \omega, 13 / 8 \omega$, and $5 / 2 \omega$. When $\omega \geq 1000 \mathrm{~Hz}$, except for the first-order frequency $\omega$, all the constituents of the frequencies vanish and the vibration may be stable.

The following figures are the time domain response graph, frequency spectrum plot, displacement-velocity phase diagram, and Poincaré section of the vibration of the pressure pipe segment in several pulse frequencies which had been shown in Figure 8.

When the pulse frequency is equal to $325 \mathrm{~Hz}$ in Figure 9, the curve of time domain response has high harmonic components. The components found in the frequency spectrum plot are $\omega, 3 \omega, 7 \omega, 9 \omega$, and $11 \omega$. The curve in $s-v$ phase diagram constitutes five closed shapes. There is only one point in Poincaré section and it can be deduced that the vibration is a periodic motion.

Under $\omega=425 \mathrm{~Hz}$ in Figure 10, the response curve has many local peaks and it is constituted by lots of high and low frequencies, as shown in frequency spectrum plot, $3 \omega$, $5 \omega, 3 / 2 \omega, 5 / 2 \omega, 7 / 2 \omega, 9 / 2 \omega$, and many other combinations. The $s-v$ phase diagram is made up by many closed curves combining with Poincaré section, and it can be indicated that the response of pipe wall is the quasiperiodic motion.

From Figure 11, the time domain response curve displays two local peaks, a high-amplitude peak and a smallamplitude one. Two obvious frequencies can be seen in frequency spectrum plot. Combining $s-v$ phase diagram and Poincaré section, it can be got that the response is the periodic motion at $\omega=775 \mathrm{~Hz}$.

As shown in Figure 12, when $\omega=1000 \mathrm{~Hz}$, the time waveform curve has many different peaks. In the frequency spectrum plot there are four obvious peaks $1 / 3 \omega, 3 / 8 \omega, \omega$, and $13 / 8 \omega$ and there are also a lot of frequency ingredients whose amplitudes are very small. The $s-v$ phase diagram is made up of many closed curve and the Poincaré section consists of a large number of points lying on a closed curve. These all showed that the response of pipe wall is the quasiperiodic motion.

From Figure 13, at the pulse frequency $\omega=1200 \mathrm{~Hz}$, the steady time domain response curve approximates to a sine or a cosine curve. And only one frequency, which is the pulse frequency, can be seen in the frequency spectrum plot. 


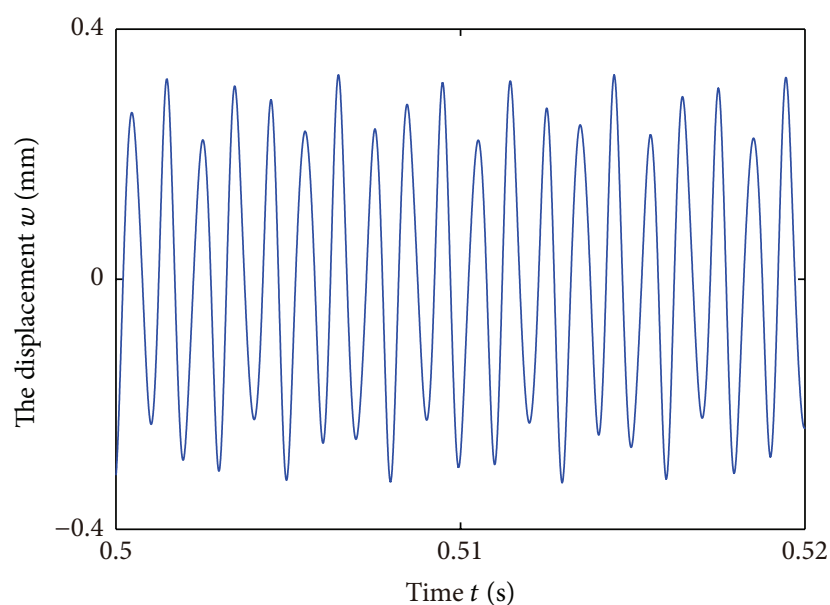

(a) Time domain response graph

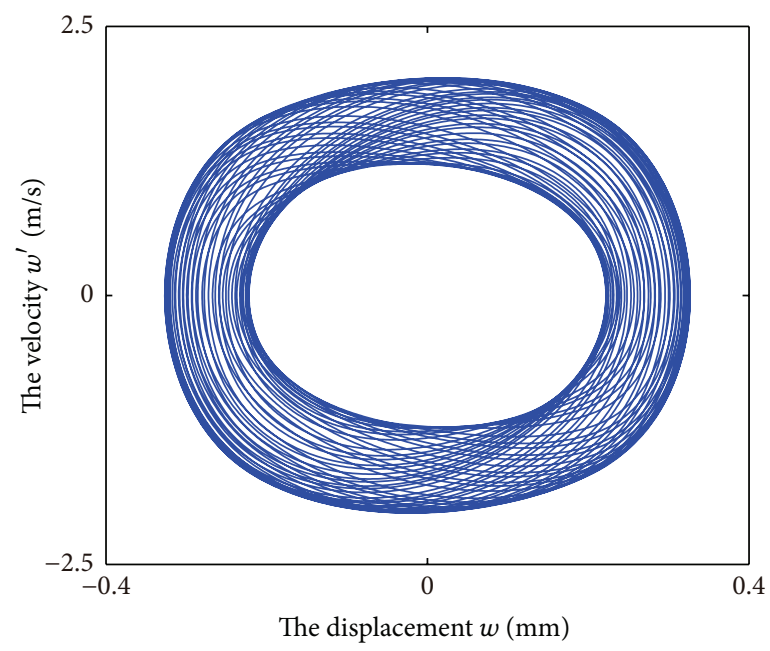

(c) $s-v$ phase diagram

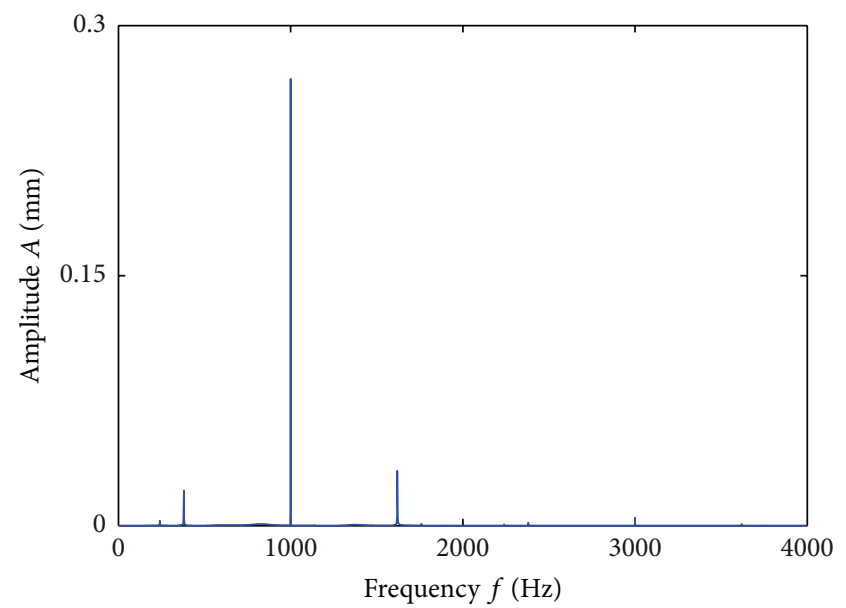

(b) Frequency spectrum plot

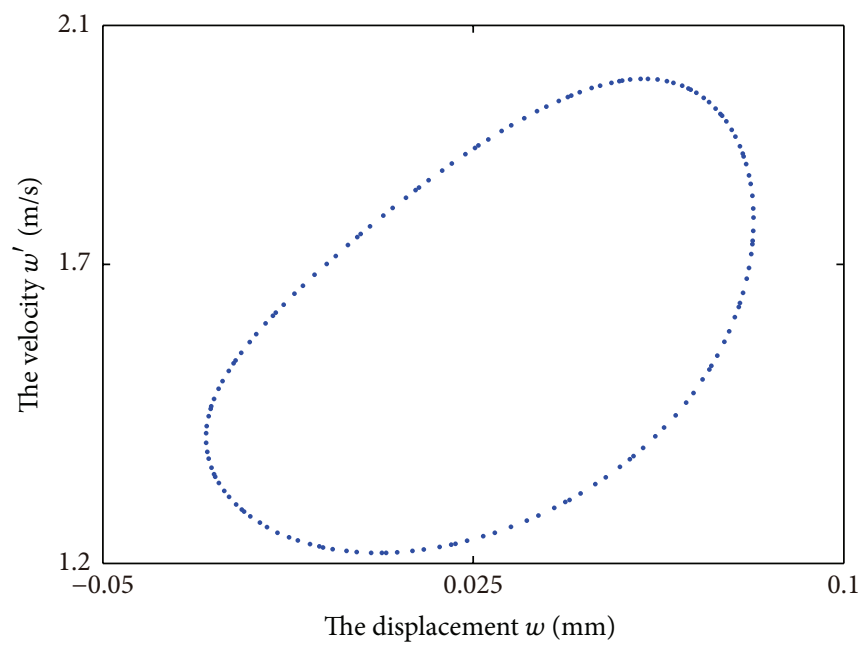

(d) Poincaré section

FIGURE 17: Kinetic characteristic curve under the condition $k=4 \times 10^{8} \mathrm{~N} / \mathrm{m}$.

The $s-v$ phase diagram is a smooth oval and there is only one point in the Poincaré section. These all indicate that the vibration response of pipe wall is a periodic motion.

\subsection{Effect of Supporting Stiffness on Dynamic Response of} Pressure Pipe Segment. Supporting stiffness is an important parameter for dynamic response of pressure pipe segment vibration. Different supporting stiffness would cause different dynamic behaviors. In this part, dynamic behaviors of pressure pipe segment would be analyzed by changing the value of supporting stiffness and keeping other parameters unchanged. The dimensional spectrum plots and bifurcation diagrams were given to study the effect of stiffness on dynamic characteristic and stability of pressure pipe segment. And then time domain response graph, frequency spectrum plot, displacement-velocity phase diagram, and Poincaré section were selected as tools to analyze in detail the nonlinear dynamic behaviors of the pipe wall vibration under several supporting stiffness values.

Figure 14 gives the dimensional spectrums of pipe wall under the stiffness $1 \times 10^{8} \mathrm{~N} / \mathrm{m}, 5 \times 10^{8} \mathrm{~N} / \mathrm{m}$, and
$1 \times 10^{9} \mathrm{~N} / \mathrm{m}$, respectively. By comparison of these three pictures, the stiffness has an important effect on the components and amplitudes of response frequencies. For example, as shown in the figure, at the stiffness $k=1 \times 10^{8} \mathrm{~N} / \mathrm{m}$, the obvious frequencies are $\omega$ and $3 \omega$. Other frequencies $5 \omega$ and $7 \omega$ occur in low order pulse frequency region and their amplitudes are very small. The frequency components become abundant when the stiffness $k=5 \times 10^{8} \mathrm{~N} / \mathrm{m}$. There are not only $\omega, 2 \omega, 3 \omega, 4 \omega, 5 \omega$, and $7 \omega$, but also $1 / 3 \omega$ and $1 / 4 \omega$ and many combinations of frequency multiplication and frequency de multiplication. It can be found compared to $k=1 \times 10^{8} \mathrm{~N} / \mathrm{m}$ that the amplitude of $\omega$ decreases and the amplitudes of $2 \omega, 3 \omega, 5 \omega$, and $7 \omega$ increase. This indicated that more frequencies make a significant contribution to the vibration energy. The nonlinearity is more complex when $k=1 \times 10^{9} \mathrm{~N} / \mathrm{m}$, the frequency components found in Figure 14(c) are various, and the amplitudes of some frequency multiplication are quite large. Continuous broadband random spectrum appeared under some frequencies. All the frequency components have a significative contribution to the vibrational energy. Therefore, the system would show 


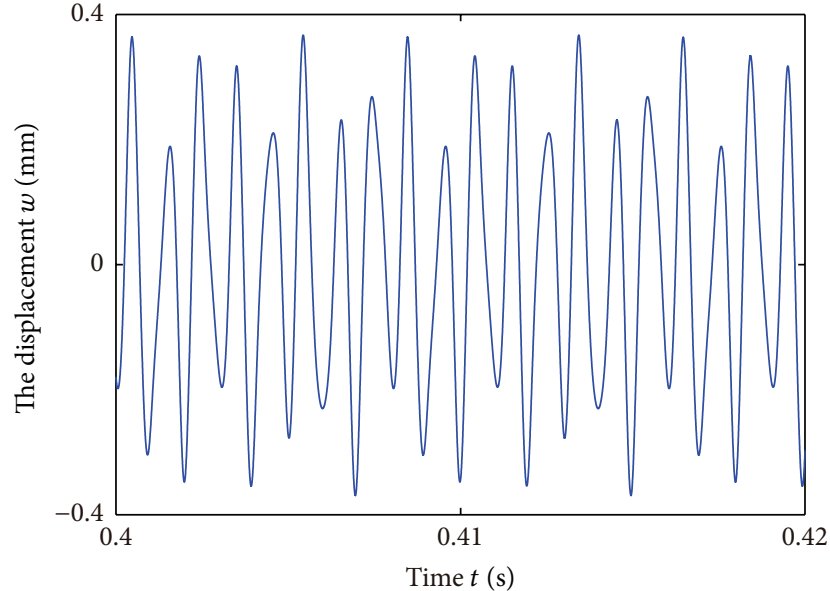

(a) Time domain response graph

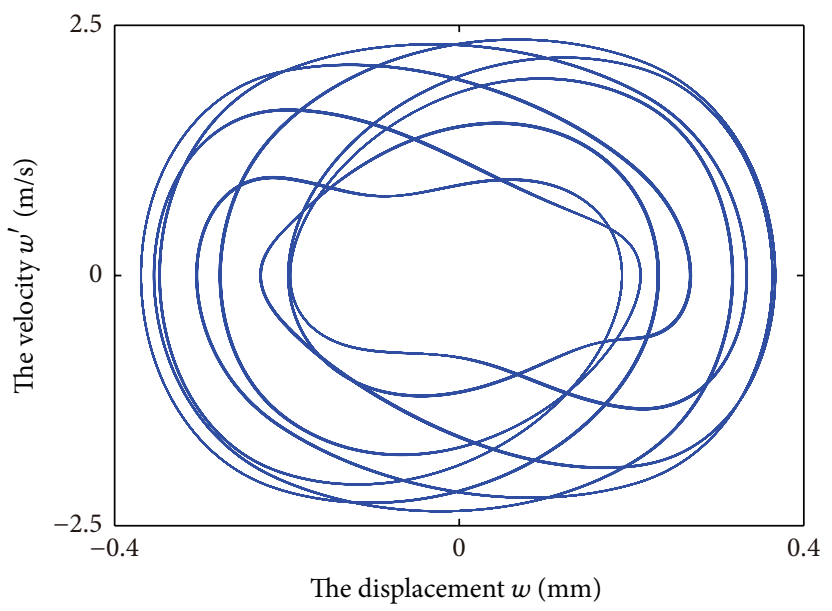

(c) $s-v$ phase diagram

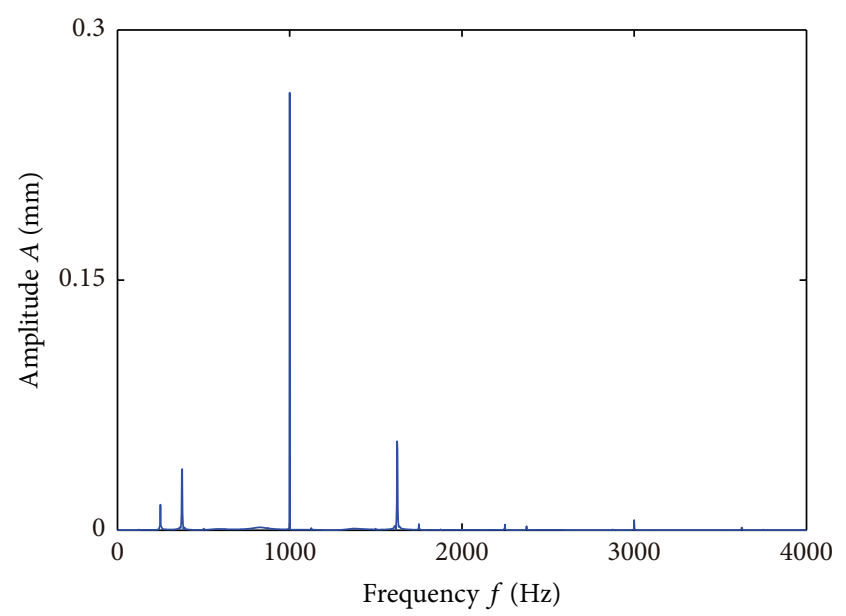

(b) Frequency spectrum plot

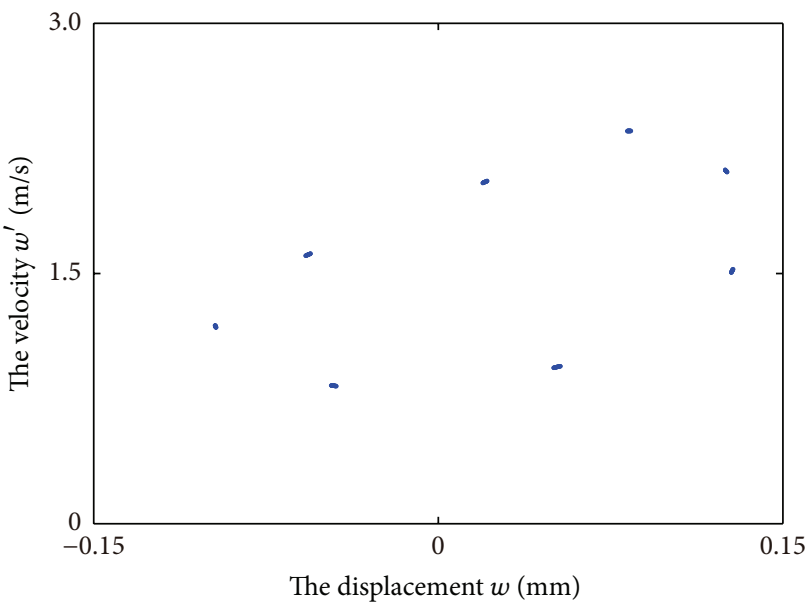

(d) Poincaré section

FIGURE 18: Kinetic characteristic curve under the condition $k=4.7 \times 10^{8} \mathrm{~N} / \mathrm{m}$.

different nonlinear behaviors under different supporting stiffness.

To explain the dynamic behaviors in detail, arranging supporting stiffness as bifurcation parameter, which is in the range from $1 \times 10^{7} \mathrm{~N} / \mathrm{m}$ to $10 \times 10^{8} \mathrm{~N} / \mathrm{m}$, the dimensional spectrum plots and bifurcation diagrams were obtained as Figure 15 at the condition of $\omega=1000 \mathrm{~Hz}$ and $b=0.2 \mathrm{~mm}$.

As demonstrated in Figure 15, when supporting stiffness is less than $4 \times 10^{8} \mathrm{~N} / \mathrm{m}$, the main frequencies are $\omega$ and $3 \omega$, and $3 \omega$ 's amplitude is far less than $\omega$, and their amplitudes have a little change as the stiffness varies in the range from $1 \times 10^{7} \mathrm{~N} / \mathrm{m}$ to $4 \times 10^{8} \mathrm{~N} / \mathrm{m}$. By combination with bifurcation diagram, in which the curve is a straight line, the response may be a stable period motion. When the stiffness $4 \times$ $10^{8} \mathrm{~N} / \mathrm{m}<k<9.5 \times 10^{8} \mathrm{~N} / \mathrm{m}, 1 / 8 \omega, 1 / 4 \omega, 3 / 8 \omega, \omega, 9 / 8 \omega$, $3 / 2 \omega, 13 / 8 \omega, 7 / 4 \omega, 5 / 2 \omega, 19 / 8 \omega, 3 \omega$, and $29 / 8 \omega$ start to emerge in the three-dimensional spectrum plot. And from the bifurcation diagram, it can be speculated that the response of pipe wall would be alternately in quasiperiodic motion, multiperiod motion, and even chaos motion. It should be noted that the loading frequency approaches the integer times of natural frequency as the stiffness increases, which causes frequency capture phenomenon. When $k>9.5 \times 10^{8} \mathrm{~N} / \mathrm{m}$, the amplitudes of $5 / 3 \omega$ and $5 / 3 \omega$ suddenly increase and that of $\omega$ suddenly decreases. It should be pointed that continuous broadband random spectrum appears beside the resonance frequencies. Seen from Figure 15(b), the response may be chaos motion.

Different dynamic behaviors of pipe wall are shown in Figures $16-19$ at $k=3 \times 10^{8} \mathrm{~N} / \mathrm{m}, k=4 \times 10^{8} \mathrm{~N} / \mathrm{m}$, $k=4.7 \times 10^{8} \mathrm{~N} / \mathrm{m}$, and $k=9.5 \times 10^{8} \mathrm{~N} / \mathrm{m}$ in the form of time domain response graph, frequency spectrum plot, $s$ $v$ phase diagram, and Poincaré section. As demonstrated in Figure 16 , when $k=3 \times 10^{8} \mathrm{~N} / \mathrm{m}$, the response curve approximates to a sine curve, and only $\omega$ is shown in frequency spectrum plot, the oval in $s-v$ phase diagram, and the only point in Poincaré section tells that the response is a period motion.

When $k=4 \times 10^{8} \mathrm{~N} / \mathrm{m}$, the response curve has many local peaks, and lots of frequencies can be found in frequency spectrum plot. The $s-v$ phase diagram is a ring composed of many ovals. The points in Poincaré section focus on an oval. 


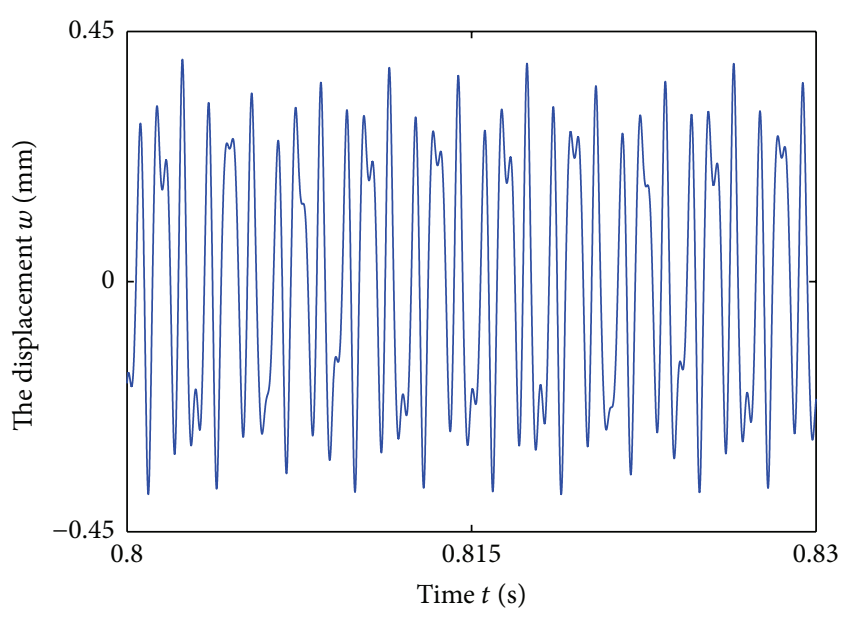

(a) Time domain response graph

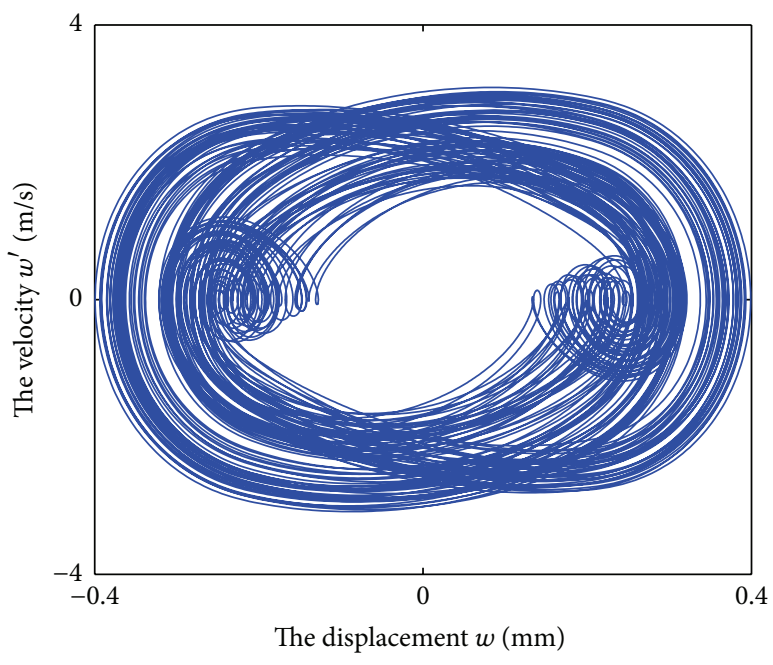

(c) $s-v$ phase diagram

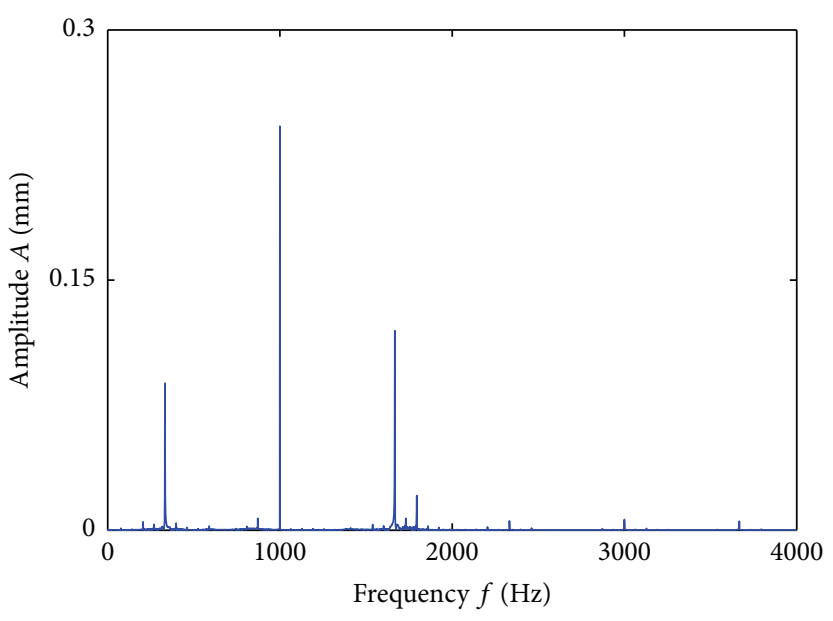

(b) Frequency spectrum plot

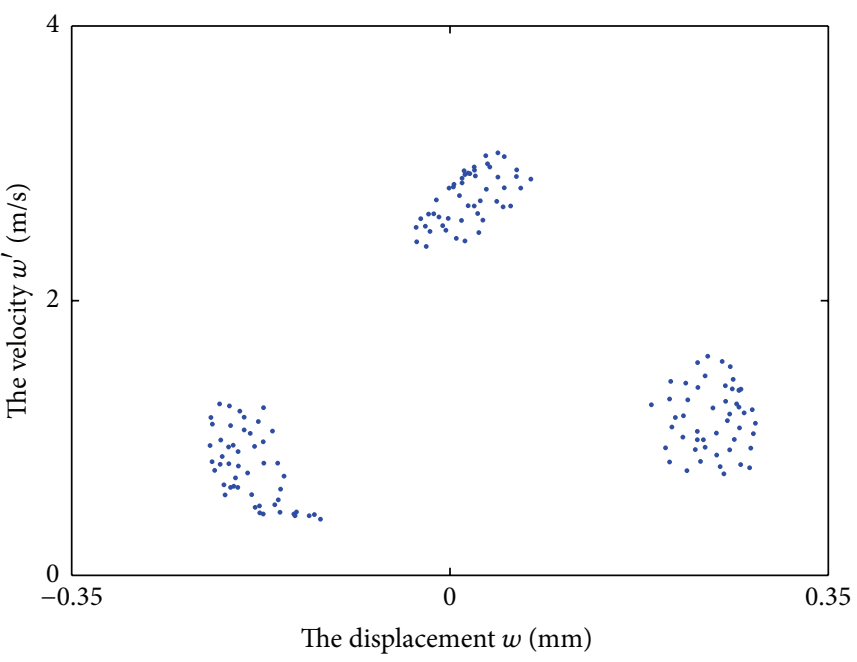

(d) Poincaré section

FIGURE 19: Kinetic characteristic curve under the condition $k=9.5 \times 10^{8} \mathrm{~N} / \mathrm{m}$.

Combining these four figures, it can be indicated that the response of pipe wall is the quasiperiodic motion.

Eight local peaks can be found in the response curve under the condition $k=4.7 \times 10^{8} \mathrm{~N} / \mathrm{m}$. Seen from frequency spectrum plot, the main frequencies are $1 \omega, 13 / \omega, 1 / 4$, and $3 / 8 \omega$. The eight closed curve in $s-v$ phase diagram and eight separate points in Poincaré section show that the response of pipe wall is the $8 T$ periodic motion.

When $k=9.5 \times 10^{8} \mathrm{~N} / \mathrm{m}$, many random local peaks appear in the response curve. Three large amplitude frequencies $1 / 3 \omega$, $\omega$, and $5 / 3 \omega$ and continuous broadband random spectrum emerge in frequency spectrum plot. The points in Poincare section mainly concentrate upon three regions, which may be the attractor phenomenon. It can be deduced that the response of pipe wall is the chaotic motion.

\subsection{Effect of Supported Clearance on Dynamic Response} of Pressure Pipe Segment. Supported clearance is also an important influence factor on dynamic response of pressure pipe segment vibration. In this part, the supported clearance was set to be the variable parameter to study dynamic behaviors of pressure pipe segment. The bifurcation diagram and the dimensional spectrum diagram, time domain response graph, frequency spectrum plot, displacement-velocity phase diagram, and Poincaré section were selected as tools to analyze in detail the nonlinear dynamic behavior of the pipe wall vibration at several supported clearance values.

Figure 20 shows the dimensional spectrum diagrams under the supported clearance $b=0.3 \mathrm{~mm}, b=0.4 \mathrm{~mm}$, and $b=0.45 \mathrm{~mm}$, respectively. By comparison of these three pictures, it is easily found that the supported clearance has a great effect on frequency component and amplitude of pipe response, especially in some sensitive region of pulse frequency. For example, the some frequency demultiplication and frequency multiplication emerge at $\omega=880 \mathrm{~Hz}$ when $b=0.3 \mathrm{~mm}$ and $b=0.4 \mathrm{~mm}$, but when $b=0.45 \mathrm{~mm}$, they disappear and the frequency $2 \omega$ at $860 \mathrm{~Hz}$ appears instead.

To analyze the form of motion, the bifurcation diagram about supported clearance is shown in Figure 21, which can be divided into four regions as the four dotted boxes. 


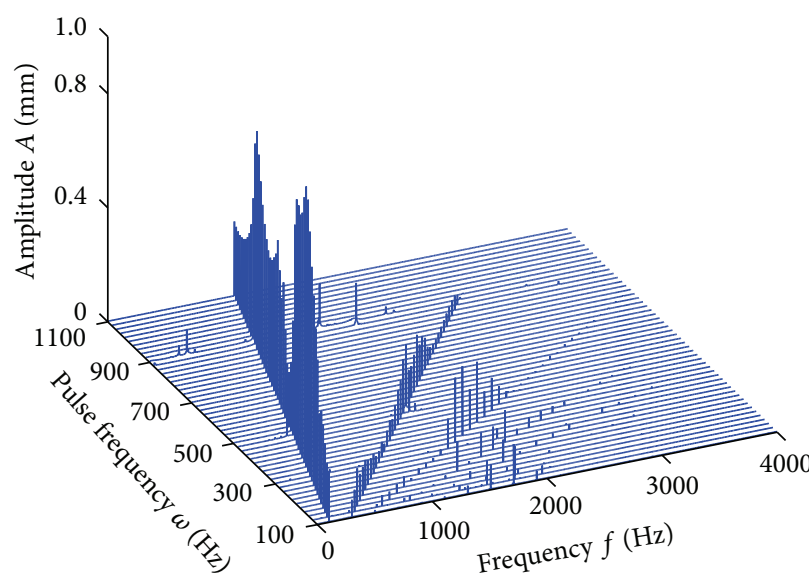

(a) $b=0.3 \mathrm{~mm}$

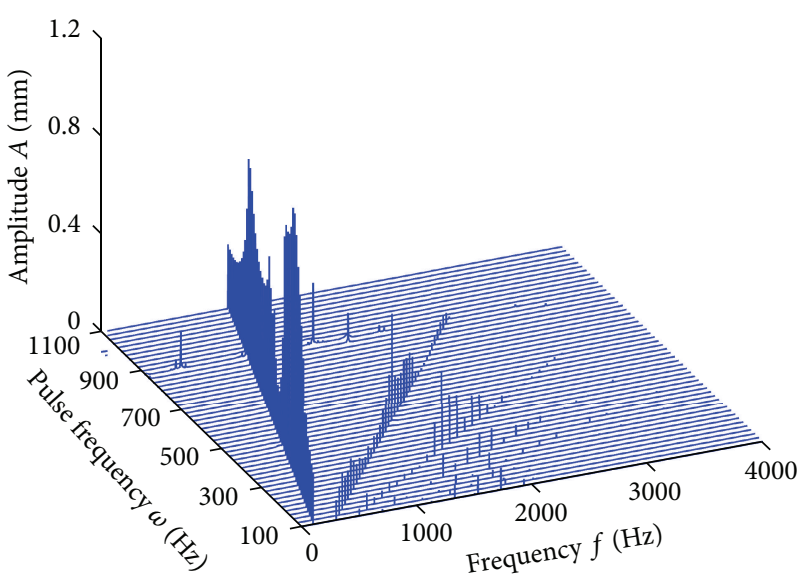

(b) $b=0.4 \mathrm{~mm}$

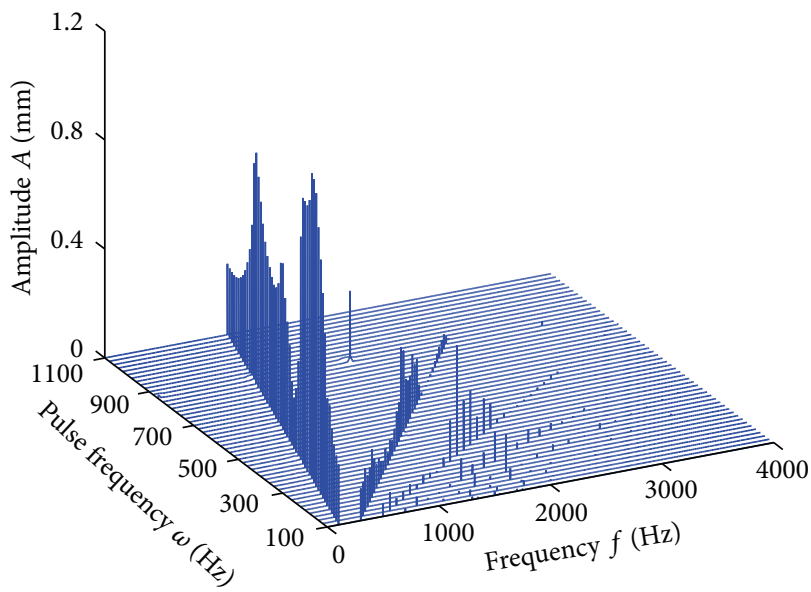

(c) $b=0.45 \mathrm{~mm}$

FIgURE 20: The three-dimensional spectrum diagram under different supported clearance.

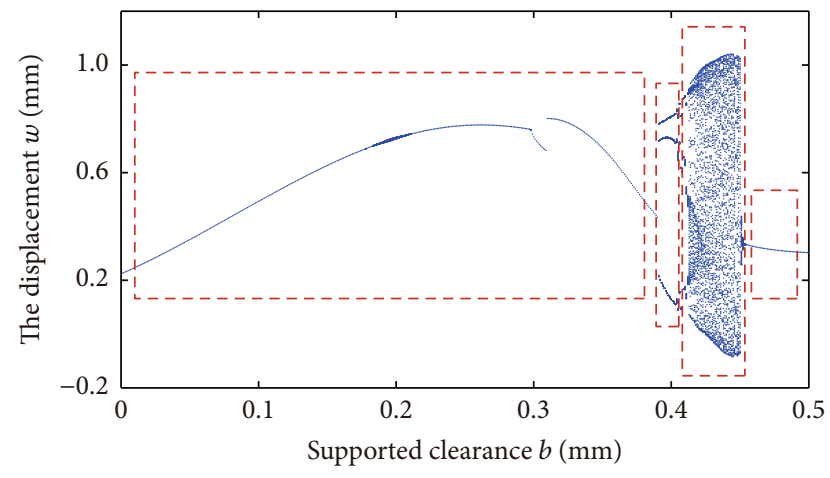

FIgURE 21: The bifurcation diagram about supported clearance.

The curve is one line when $0 \mathrm{~mm} \leq b \leq 0.39 \mathrm{~mm}$. It should be noted that jumping phenomenon happens at $b=0.31 \mathrm{~mm}$. The response may be a periodic motion in the range of supported clearance from $0 \mathrm{~mm}$ to $0.39 \mathrm{~mm}$. There appears three times bifurcation in $0.391 \mathrm{~mm} \leq b \leq 0.404 \mathrm{~mm}$, in which the response may be $3 T$-periodic motion. When $0.404 \mathrm{~mm} \leqslant b \leqslant 0.454 \mathrm{~mm}$, the bifurcation would be complex and it would be times bifurcation or chaos phenomenon indicating the motion may be the quasiperiodic motion or a chaos motion. And when $b>0.454 \mathrm{~mm}$, the curve returns to a straight line and may be returned to a periodic motion. To verify the form of motion according to Figure 21, the time domain response graph, frequency spectrum plot, displacement-velocity phase diagram, and Poincaré section at some special values of $b$ were presented. Figures 22, 23, and 24 show the dynamic behaviors of pipe segment with different clearance values.

At the supported clearance $b=0.3 \mathrm{~mm}$, the time waveform approximates to a sine or cosine curve. Frequency of $\omega, 2 \omega$, and $3 \omega$ can be seen in frequency spectrum plot. The $s-v$ phase diagram is a concave oval and there is only one point in the Poincare section. These all indicate that the vibration response of pipe wall is a periodic motion.

When $b=0.4 \mathrm{~mm}$, the peaks of time waveform have three different amplitudes. And it can be deduced that the response is compound by three harmonic components, which are shown in Figure 23(b) as $\omega, 2 \omega$ and $1 / 3 \omega$. The curve consisting of three closed curves in the $s-v$ phase diagram and the 3 isolated points in the Poincare section confirm that the response is indeed $3 T$-periodic motion. 


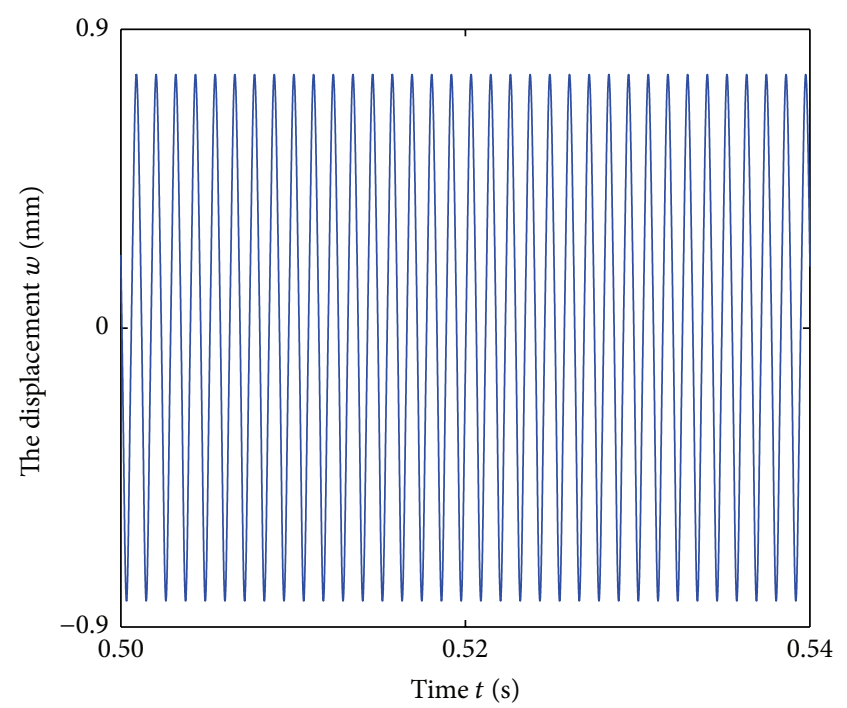

(a) Time domain response graph

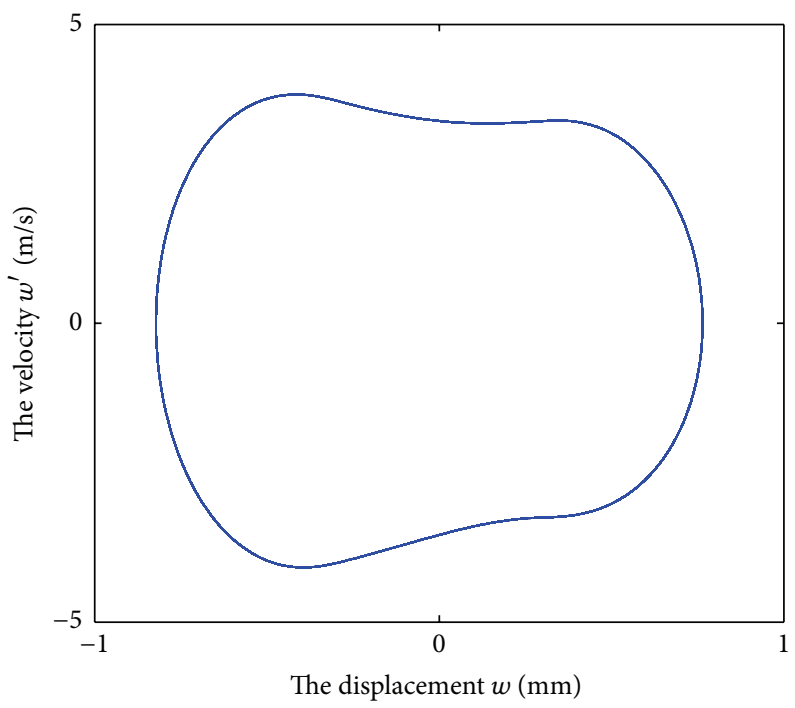

(c) $s-v$ phase diagram

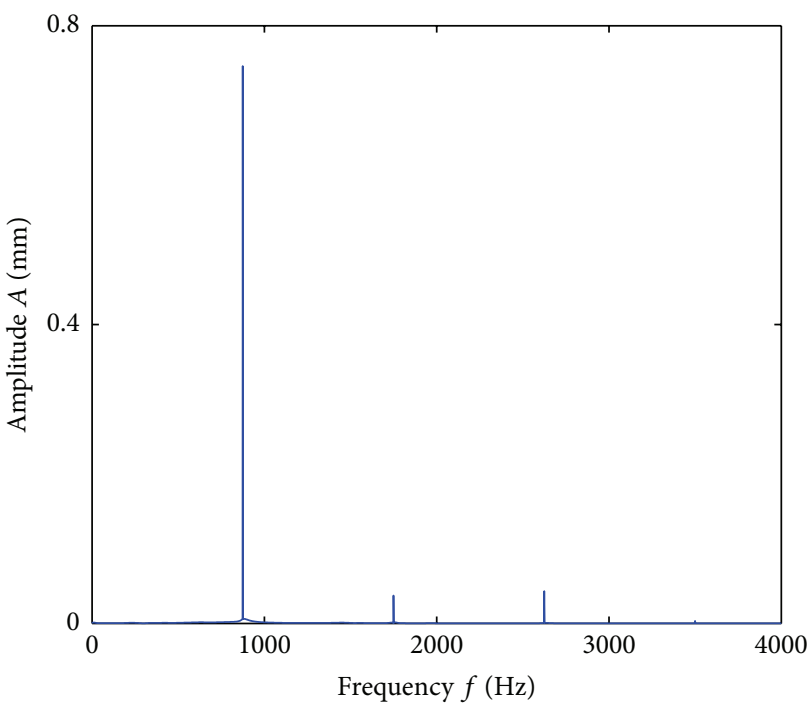

(b) Frequency spectrum plot

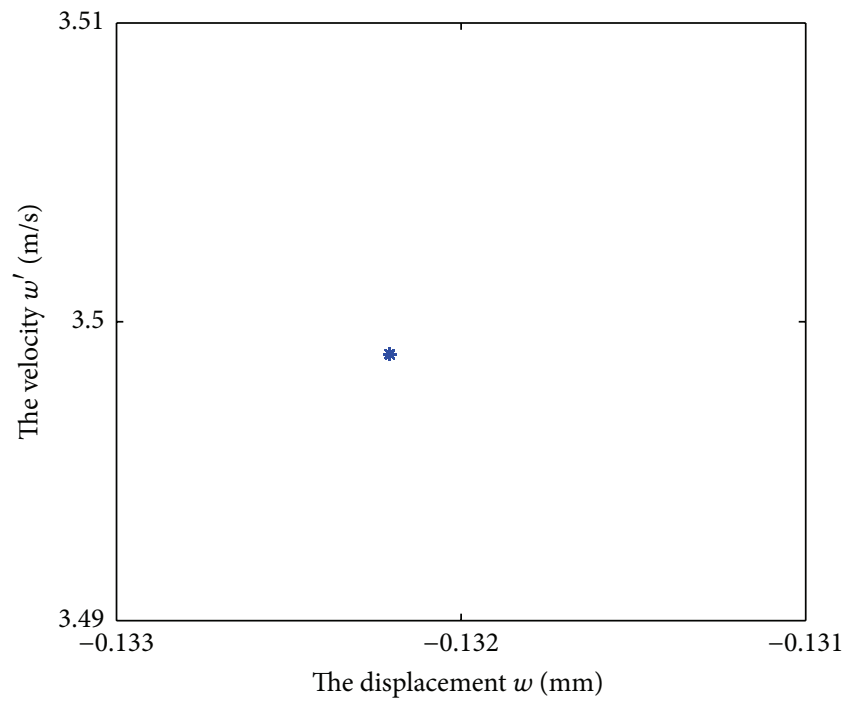

(d) Poincaré section

FIgURE 22: Kinetic characteristic curve under the condition $b=0.3 \mathrm{~mm}$.

The steady time domain response curve has many random peaks under $b=0.45 \mathrm{~mm}$. Random spectrum band appears beside the main frequencies shown in Figure 24(b). The $s-v$ phase diagram and Poincare section tell that the response of pipe wall is a chaos motion.

\section{Conclusion}

The nonlinear dynamic behaviors of pressure pipe segment with supported clearance at both ends were presented. Lagrange's equations had been written for the nonlinear vibration cases and by numerical calculation, threedimensional spectrum, bifurcation diagram, time domain response graph, frequency spectrum plot, $s-v$ phase diagram, and Poincaré section with different parameters that were obtained to analyze the effects of pulse frequency, supporting stiffness, and supported clearance on dynamic characteristics of pressure pipe. Conclusions are as follows.
By numerical simulation and calculation, the dynamics behaviors of pressure pipe segment with supported clearance at both ends show strong nonlinearity. Firstly, the amplitudefrequency curves under supported clearance skew to the right, which is called "hardening curve." Supported clearance and supporting stiffness have a great effect on resonance amplitude and resonance frequency. The larger the value of supported clearance is, the larger the resonance amplitude is and the smaller the resonance frequency is. But the nonlinearity is more obvious under smaller clearance. The high harmonic which has the large amplitude indicates that high-order resonance may happen under the condition with supported clearance. Jump phenomena emerge obviously at some frequencies in the process of frequency change. They would cause large relative motion, which may produce so great stress that fatigue failure happens to reduce the working life of pipe. Secondly, the response is also very complex. The frequency components of the pipe response 


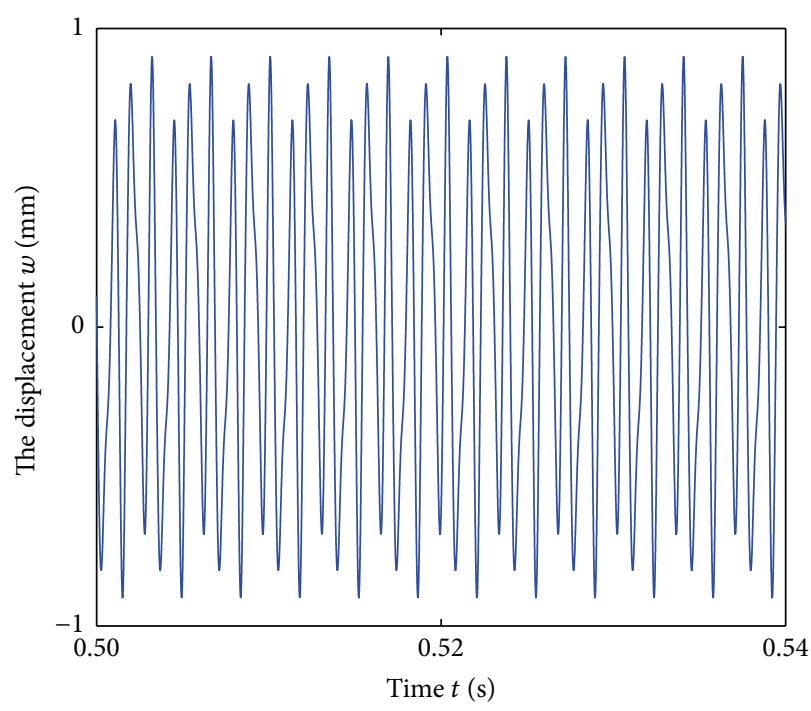

(a) Time domain response graph

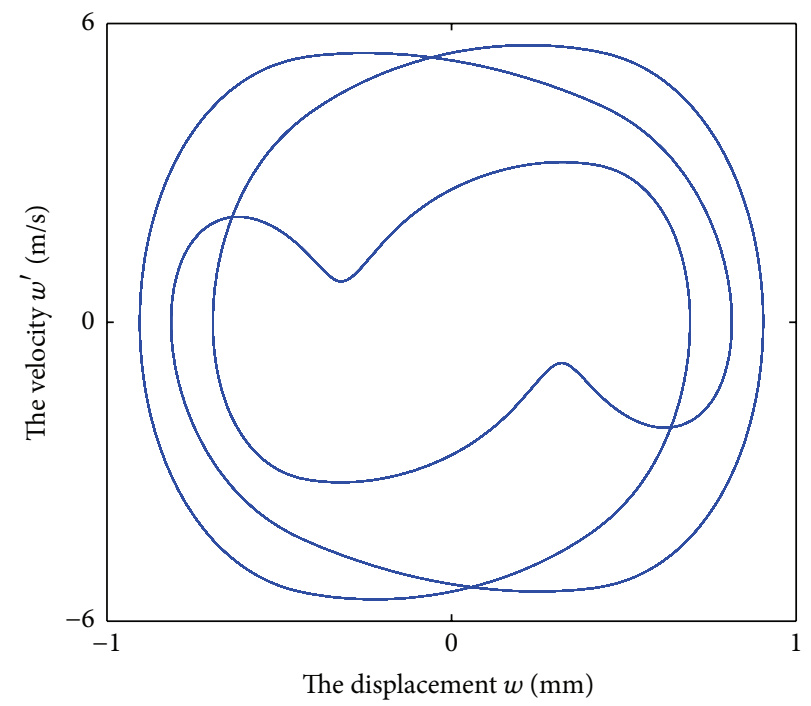

(c) $s$ - $v$ phase diagram

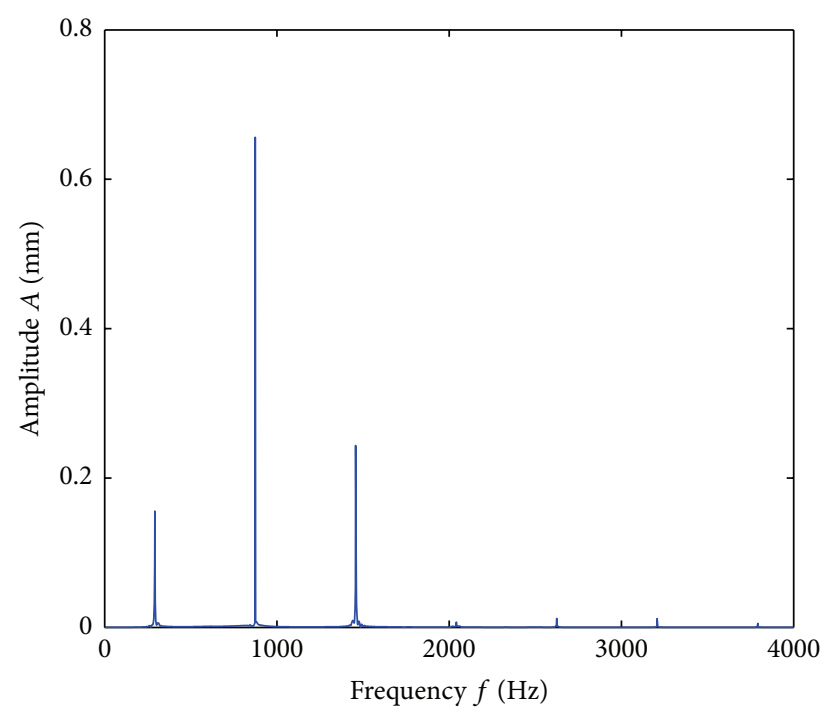

(b) Frequency spectrum plot

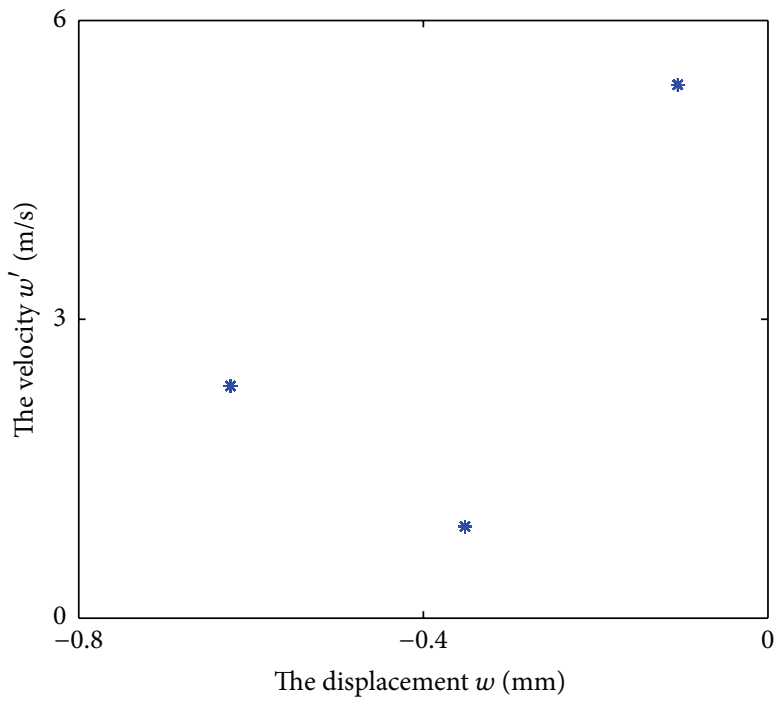

(d) Poincaré section

FIgURE 23: Kinetic characteristic curve under the condition $b=0.4 \mathrm{~mm}$.

are very abundant and they are effected by pulse frequency, supporting stiffness, and supported clearance. The complex response causes the diversification of vibration mode of pipe segment. It can be deduced from the response curve that the response of pipe segment may be the periodic, quasiperiodic, or even chaotic motion under different parameters.

\section{Appendix}

\section{A. Gram-Schmidt Orthogonalization Process of Polynomials}

$\psi_{1}^{u}(\xi), \psi_{1}^{v}(\xi)$, and $\psi_{1}^{w}(\xi)$ are given as the first terms of the polynomials in the process of generating characteristic orthogonal polynomials $\varphi_{i}^{u}(\xi), \varphi_{i}^{v}(\xi)$, and $\varphi_{i}^{w}(\xi)$ using
Gram-Schmidt method and they are satisfied according to boundary condition. $\psi_{1}^{u}(\xi)$ is applied to generate $\varphi_{i}^{u}(\xi)$ as an example:

$$
\begin{aligned}
\psi_{2}^{u}(\xi) & =\left(\xi-B_{1}^{u}\right) \psi_{1}^{u}(\xi), \\
B_{1}^{u} & =\frac{\int_{0}^{1} \xi\left[\psi_{1}^{u}(\xi)\right]^{2} \mathrm{~d} \xi}{\int_{0}^{1}\left[\psi_{1}^{u}(\xi)\right]^{2} \mathrm{~d} \xi}, \\
\psi_{k+1}^{u}(\xi) & =\left(\xi-B_{k}^{u}\right) \psi_{k}^{u}(\xi)-C_{k} \psi_{k-1}^{u}(\xi), \quad k \geq 2, \\
B_{k}^{u} & =\frac{\int_{0}^{1} \xi\left[\psi_{k}^{u}(\xi)\right]^{2} \mathrm{~d} \xi}{\int_{0}^{1}\left[\psi_{k}^{u}(\xi)\right]^{2} \mathrm{~d} \xi},
\end{aligned}
$$




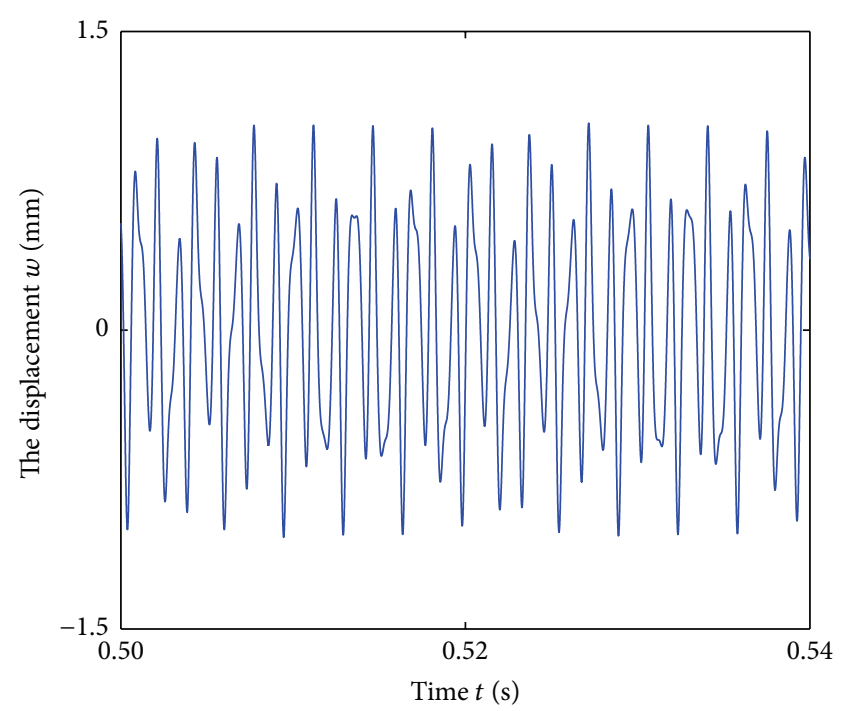

(a) Time domain response graph

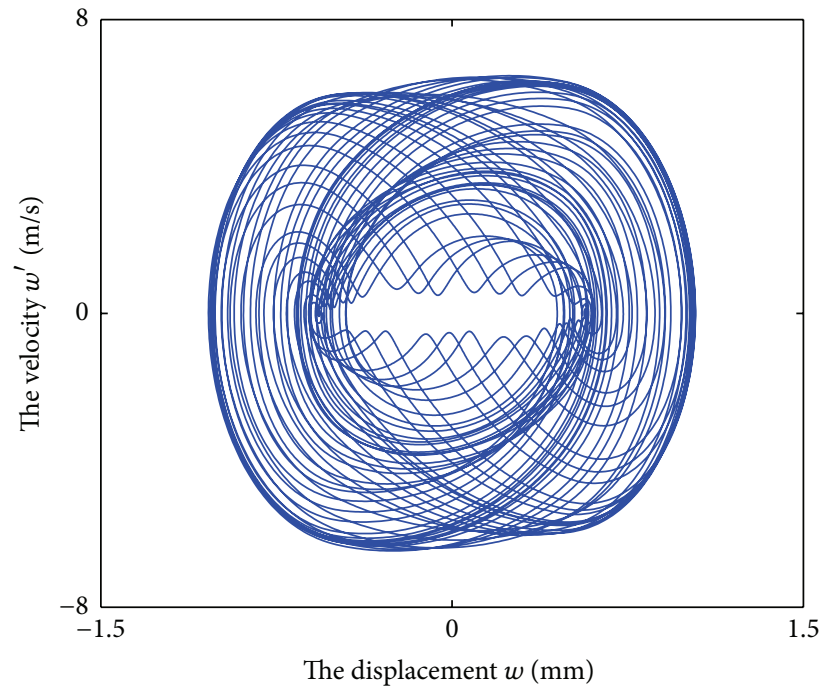

(c) $s-v$ phase diagram

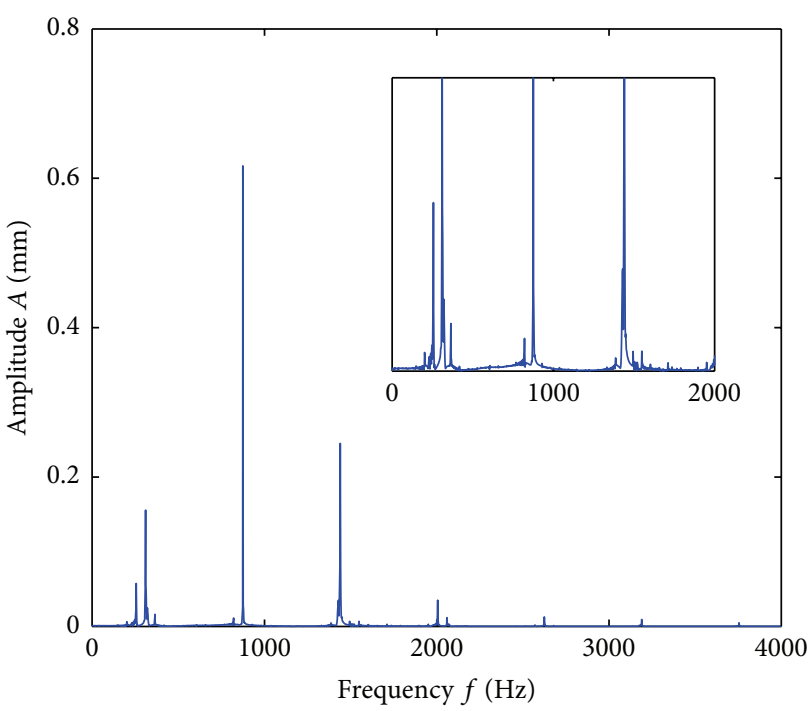

(b) Frequency spectrum plot

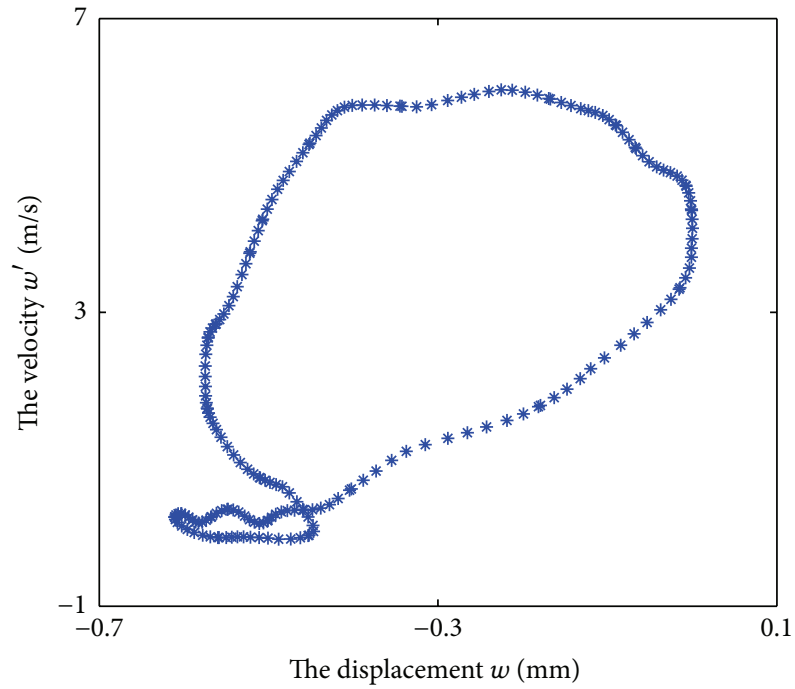

(d) Poincaré section

FIgURE 24: Kinetic characteristic curve under the condition $b=0.45 \mathrm{~mm}$.

$$
\begin{aligned}
C_{k} & =\frac{\int_{0}^{1} \xi \psi_{k}^{u}(\xi) \psi_{k-1}^{u}(\xi) \mathrm{d} \xi}{\int_{0}^{1}\left[\psi_{k-1}^{u}(\xi)\right]^{2} \mathrm{~d} \xi}, \\
\varphi_{k}^{u}(\xi) & =\frac{\psi_{k}^{u}(\xi)}{\int_{0}^{1}\left[\psi_{k}^{u}(\xi)\right]^{2} \mathrm{~d} \xi} .
\end{aligned}
$$

It should be noted that the polynomials constructed satisfy the orthogonality condition:

$$
\int_{0}^{1} \xi \varphi_{k}^{u}(\xi) \varphi_{l}^{u}(\xi) \mathrm{d} \xi= \begin{cases}0, & k \neq l \\ 1, & k=l\end{cases}
$$

The same operation can be done for $\psi_{1}^{v}(\xi)$ and $\psi_{1}^{w}(\xi)$.

\section{B. Formulations for Elements in (10), (11), and (12)}

One has the following:

$\mathbf{M}_{1}=\rho h L \int_{0}^{1} \int_{0}^{2 \pi} \mathbf{U} \mathbf{U}^{\mathrm{T}} R \mathrm{~d} \theta \mathrm{d} \xi$,

$\mathbf{M}_{2}=\rho h L \int_{0}^{1} \int_{0}^{2 \pi} \mathbf{V V}^{\mathrm{T}} R \mathrm{~d} \theta \mathrm{d} \xi$,

$\mathbf{M}_{3}=\rho h L \int_{0}^{1} \int_{0}^{2 \pi} \mathbf{W W}^{\mathrm{T}} R \mathrm{~d} \theta \mathrm{d} \xi$,

$\mathbf{K}_{1}=\int_{0}^{1} \int_{0}^{2 \pi}\left\{\frac{E h}{\left(1-\mu^{2}\right)} \frac{1}{L^{2}} \frac{\partial \mathbf{U}}{\partial \xi} \frac{\partial \mathbf{U}^{\mathrm{T}}}{\partial \xi}\right.$ 
$\left.+\left[\frac{E h}{\left(1-\mu^{2}\right)} \frac{1-\mu}{2} \frac{1}{R^{2}}+\frac{E h^{3}}{12\left(1-\mu^{2}\right)} \frac{(1-\mu)}{2 R^{2}} \frac{1}{4 R^{2}}\right] \frac{\partial \mathbf{U}}{\partial \theta} \frac{\partial \mathbf{U}^{\mathrm{T}}}{\partial \theta}\right\}$

$\cdot R L \mathrm{~d} \theta \mathrm{d} \xi$

$$
\begin{aligned}
\mathbf{K}_{2} & =\int_{0}^{1} \int_{0}^{2 \pi} \frac{E h}{\left(1-\mu^{2}\right)} \frac{2 \mu}{R L} \frac{\partial \mathbf{U}}{\partial \xi} \frac{\partial \mathbf{V}^{\mathrm{T}}}{\partial \theta}+\left[\frac{E h}{\left(1-\mu^{2}\right)} \frac{1-\mu}{2} \frac{2}{R L}\right. \\
& -\frac{E h^{3}}{12\left(1-\mu^{2}\right)} \frac{(1-\mu)}{2 R^{2}} \\
& \left.\cdot \frac{3}{2 R L}\right] \frac{\partial \mathbf{U}}{\partial \theta} \frac{\partial \mathbf{V}^{\mathrm{T}}}{\partial \xi} R L \mathrm{~d} \theta \mathrm{d} \xi,
\end{aligned}
$$$$
\mathbf{K}_{3}=\int_{0}^{1} \int_{0}^{2 \pi}\left[\frac{E h}{\left(1-\mu^{2}\right)} \frac{2 \mu}{R L} \frac{\partial \mathbf{U}}{\partial \xi} \mathbf{W}^{\mathrm{T}}+\frac{E h^{3}}{12\left(1-\mu^{2}\right)} \frac{(1-\mu)}{2 R^{2}} \frac{2}{R L}\right.
$$$$
\left.\frac{\partial \mathbf{U}}{\partial \theta} \frac{\partial^{2} \mathbf{W}^{\mathrm{T}}}{\partial \xi \partial \theta}\right] R L \mathrm{~d} \theta \mathrm{d} \xi
$$$$
\begin{aligned}
\mathbf{K}_{4} & =\int_{0}^{1} \int_{0}^{2 \pi}\left\{\left[\frac{E h}{\left(1-\mu^{2}\right)} \frac{1}{R^{2}}+\frac{E h^{3}}{12\left(1-\mu^{2}\right)} \frac{1}{R^{4}}\right] \frac{\partial \mathbf{V}}{\partial \theta} \frac{\partial \mathbf{V}^{\mathrm{T}}}{\partial \theta}\right. \\
& \left.+\left[\frac{E h}{\left(1-\mu^{2}\right)} \frac{1-\mu}{2} \frac{1}{L^{2}}+\frac{E h^{3}}{12\left(1-\mu^{2}\right)} \frac{(1-\mu)}{2 R^{2}} \frac{9}{4 L^{2}}\right] \frac{\partial \mathbf{V}}{\partial \xi} \frac{\partial \mathbf{V}^{\mathrm{T}}}{\partial \xi}\right\}
\end{aligned}
$$

- $R L \mathrm{~d} \theta \mathrm{d} \xi$

$$
\begin{aligned}
\mathbf{K}_{5} & =\int_{0}^{1} \int_{0}^{2 \pi}\left\{\frac{E h}{\left(1-\mu^{2}\right)} \frac{2}{R^{2}} \frac{\partial \mathbf{V}}{\partial \theta} \mathbf{W}^{\mathrm{T}}-\frac{E h^{3}}{12\left(1-\mu^{2}\right)} \frac{2 \mu}{R^{2} L^{2}} \frac{\partial \mathbf{V}}{\partial \theta}\right. \\
& \cdot \frac{\partial^{2} \mathbf{W}^{\mathrm{T}}}{\partial \xi^{2}}-\frac{E h^{3}}{12\left(1-\mu^{2}\right)} \frac{2}{R^{4}} \frac{\partial \mathbf{V}}{\partial \theta} \frac{\partial^{2} \mathbf{W}^{\mathrm{T}}}{\partial \theta^{2}}-\frac{E h^{3}}{12\left(1-\mu^{2}\right)} \frac{(1-\mu)}{2 R^{2}} \\
& \left.\cdot \frac{6}{L^{2}} \frac{\partial \mathbf{V}}{\partial \xi} \frac{\partial^{2} \mathbf{W}^{\mathrm{T}}}{\partial \xi \partial \theta}\right\} R L \mathrm{~d} \theta \mathrm{d} \xi,
\end{aligned}
$$$$
\mathbf{K}_{6}=\int_{0}^{1} \int_{0}^{2 \pi}\left\{\frac{E h}{\left(1-\mu^{2}\right)} \frac{1}{R^{2}} \mathbf{W} \mathbf{W}^{\mathrm{T}}+\frac{E h^{3}}{12\left(1-\mu^{2}\right)} \frac{1}{L^{4}} \frac{\partial^{2} \mathbf{W}}{\partial \xi^{2}} \frac{\partial^{2} \mathbf{W}^{\mathrm{T}}}{\partial \xi^{2}}\right.
$$$$
+\frac{E h^{3}}{12\left(1-\mu^{2}\right)} \frac{2 \mu}{R^{2} L^{2}} \frac{\partial^{2} \mathbf{W}}{\partial \xi^{2}} \frac{\partial^{2} \mathbf{W}^{\mathrm{T}}}{\partial \theta^{2}}+\frac{E h^{3}}{12\left(1-\mu^{2}\right)} \frac{1}{R^{4}} \frac{\partial^{2} \mathbf{W}}{\partial \theta^{2}}
$$$$
\left.\cdot \frac{\partial^{2} \mathbf{W}^{\mathrm{T}}}{\partial \theta^{2}}+\frac{E h^{3}}{12\left(1-\mu^{2}\right)} \frac{(1-\mu)}{2 R^{2}} \frac{4}{L^{2}} \frac{\partial^{2} \mathbf{W}}{\partial \xi \partial \theta} \frac{\partial^{2} \mathbf{W}^{\mathrm{T}}}{\partial \xi \partial \theta}\right\} R L \mathrm{~d} \theta \mathrm{d} \xi,
$$$$
\mathbf{D}=-\int_{0}^{1} \int_{0}^{2 \pi} \frac{p(\xi) L}{2}\left[\left(\frac{\partial^{2} \mathbf{W}}{\partial \theta^{2}}+\mathbf{W}\right) \mathbf{W}^{\mathrm{T}}\right] \mathrm{d} \theta \mathrm{d} \xi .
$$

\section{Competing Interests}

The authors declare that there is no conflict of interests regarding the publication of this paper.

\section{Acknowledgments}

The project was supported by Fundamental Research Funds for the Central Universities (nos. N140304002 and
N140301001) and the China Natural Science Funds (no. 51575093).

\section{References}

[1] H. W. Lee and M. K. Kwak, "Free vibration analysis of a circular cylindrical shell using the Rayleigh-Ritz method and comparison of different shell theories," Journal of Sound and Vibration, vol. 353, pp. 344-377, 2015.

[2] K. Y. Lam and C. T. Loy, "Effects of boundary conditions on frequencies of a multi-layered cylindrical shell," Journal of Sound and Vibration, vol. 188, no. 3, pp. 363-384, 1995.

[3] C. T. Loy and K. Y. Lam, "Vibration of thick cylindrical shells on the basis of three-dimensional theory of elasticity," Journal of Sound and Vibration, vol. 226, no. 4, pp. 719-737, 1999.

[4] C. T. Loy, K. Y. Lam, and J. N. Reddy, "Vibration of functionally graded cylindrical shells," International Journal of Mechanical Sciences, vol. 41, no. 3, pp. 309-324, 1999.

[5] C. T. Loy, K. Y. Lam, and C. Shu, "Analysis of cylindrical shells using generalized differential quadrature," Shock and Vibration, vol. 4, no. 3, pp. 193-198, 1997.

[6] X. M. Zhang, G. R. Liu, and K. Y. Lam, "Vibration analysis of thin cylindrical shells using wave propagation approach," Journal of Sound and Vibration, vol. 239, no. 3, pp. 397-403, 2001.

[7] X. Xie, G. Jin, and Z. Liu, "Free vibration analysis of cylindrical shells using the Haar wavelet method," International Journal of Mechanical Sciences, vol. 77, pp. 47-56, 2013.

[8] G. Xie, "Free vibration analysis of circular cylindrical shells using transfer matrix method," in Proceedings of the International Conference on Electric Information and Control Engineering (ICEICE '11), pp. 423-426, Wuhan, China, April 2011.

[9] M. J. Ebrahimi and M. M. Najafizadeh, "Free vibration analysis of two-dimensional functionally graded cylindrical shells," Applied Mathematical Modelling, vol. 38, no. 1, pp. 308-324, 2014.

[10] S. Sun, S. Chu, and D. Cao, "Vibration characteristics of thin rotating cylindrical shells with various boundary conditions," Journal of Sound and Vibration, vol. 331, no. 18, pp. 4170-4186, 2012.

[11] A. V. Lopatin and E. V. Morozov, "Fundamental frequency of the laminated composite cylindrical shell with clamped edges," International Journal of Mechanical Sciences, vol. 92, pp. 35-43, 2015.

[12] Y. Wang, L. Liang, X. Guo, J. Li, J. Liu, and P. Liu, "Nonlinear vibration response and bifurcation of circular cylindrical shells under traveling concentrated harmonic excitation," Acta Mechanica Solida Sinica, vol. 26, no. 3, pp. 277-291, 2013.

[13] T. Dey and L. S. Ramachandra, "Dynamic stability of simply supported composite cylindrical shells under partial axial loading," Journal of Sound and Vibration, vol. 353, pp. 272-291, 2015.

[14] M. R. Isvandzibaei, H. Jamaluddin, and R. I. Raja Hamzah, "Frequency analysis of multiple layered cylindrical shells under lateral pressure with asymmetric boundary conditions," Chinese Journal of Mechanical Engineering, vol. 27, no. 1, pp. 23-31, 2014.

[15] M. R. Isvandzibaei, H. Jamaluddin, and R. I. R. Hamzah, "Natural frequency characteristics of thin-walled homogeneous and manifold layered cylindrical shells under pressure using energy method," Journal of Central South University, vol. 21, no. 2, pp. 521-532, 2014. 
[16] M. R. Isvandzibaei, H. Jamaluddin, and R. I. Raja Hamzah, "Effects of uniform interior pressure distribution on vibration of FGM cylindrical shell with rings support based on first-order theory subjected to ten boundary conditions," Acta Mechanica, vol. 225, no. 7, pp. 2085-2109, 2014.

[17] F. Forouzesh and A. A. Jafari, "Radial vibration analysis of pseudoelastic shape memory alloy thin cylindrical shells by the differential quadrature method," Thin-Walled Structures, vol. 93, pp. 158-168, 2015.

[18] G. Jin, T. Ye, Y. Chen, Z. Su, and Y. Yan, "An exact solution for the free vibration analysis of laminated composite cylindrical shells with general elastic boundary conditions," Composite Structures, vol. 106, pp. 114-127, 2013.

[19] G. Jin, C. Yang, Z. Liu, S. Gao, and C. Zhang, "A unified method for the vibration and damping analysis of constrained layer damping cylindrical shells with arbitrary boundary conditions," Composite Structures, vol. 130, pp. 124-142, 2015.

[20] G. Jin, T. Ye, X. Ma, Y. Chen, Z. Su, and X. Xie, "A unified approach for the vibration analysis of moderately thick composite laminated cylindrical shells with arbitrary boundary conditions," International Journal of Mechanical Sciences, vol. 75, pp. 357-376, 2013.

[21] Y. Qu, Y. Chen, X. Long, H. Hua, and G. Meng, "Free and forced vibration analysis of uniform and stepped circular cylindrical shells using a domain decomposition method," Applied Acoustics, vol. 74, no. 3, pp. 425-439, 2013.

[22] L. Dai, T. Yang, J. Du, W. L. Li, and M. J. Brennan, "An exact series solution for the vibration analysis of cylindrical shells with arbitrary boundary conditions," Applied Acoustics, vol. 74, no. 3, pp. 440-449, 2013.

[23] Y. Chen, G. Jin, and Z. Liu, "Free vibration analysis of circular cylindrical shell with non-uniform elastic boundary constraints," International Journal of Mechanical Sciences, vol. 74, pp. 120-132, 2013.

[24] H. Zhou, W. Li, B. Lv, and W. L. Li, "Free vibrations of cylindrical shells with elastic-support boundary conditions," Applied Acoustics, vol. 73, no. 8, pp. 751-756, 2012.

[25] D. Shi, Y. Zhao, Q. Wang, X. Teng, and F. Pang, "A unified spectro-geometric-ritz method for vibration analysis of open and closed shells with arbitrary boundary conditions," Shock and Vibration, vol. 2016, Article ID 4097123, 30 pages, 2016.

[26] S. Sun, D. Cao, and Q. Han, "Vibration studies of rotating cylindrical shells with arbitrary edges using characteristic orthogonal polynomials in the Rayleigh-Ritz method," International Journal of Mechanical Sciences, vol. 68, pp. 180-189, 2013.

[27] L. Liu, D. Cao, and S. Sun, "Vibration analysis for rotating ringstiffened cylindrical shells with arbitrary boundary conditions," Journal of Vibration and Acoustics, vol. 135, no. 6, Article ID 061010, 2013.

[28] F. Bakhtiari-Nejad and S. M. Mousavi Bideleh, "Nonlinear free vibration analysis of prestressed circular cylindrical shells on the Winkler/Pasternak foundation," Thin-Walled Structures, vol. 53, pp. 26-39, 2012.

[29] A. G. Shah, T. Mahmood, M. N. Naeem, Z. Iqbal, and S. H. Arshad, "Vibrations of functionally graded cylindrical shells based on elastic foundations," Acta Mechanica, vol. 211, no. 34, pp. 293-307, 2010.

[30] A. A. Khdeir and J. N. Reddy, "Influence of edge conditions on the modal characteristics of cross-ply laminated shells," Computers and Structures, vol. 34, no. 6, pp. 817-826, 1990. 


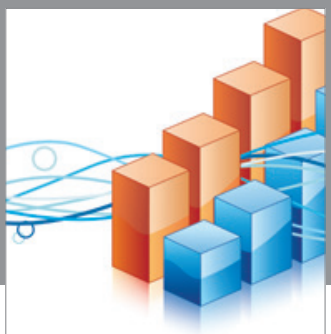

Advances in

Operations Research

vatem alat4

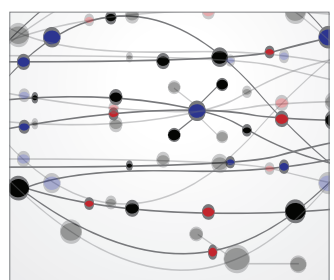

\section{The Scientific} World Journal
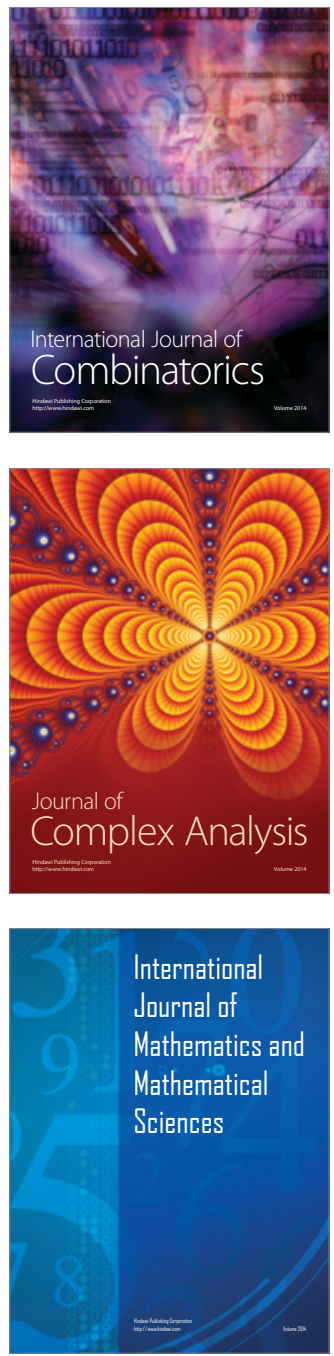
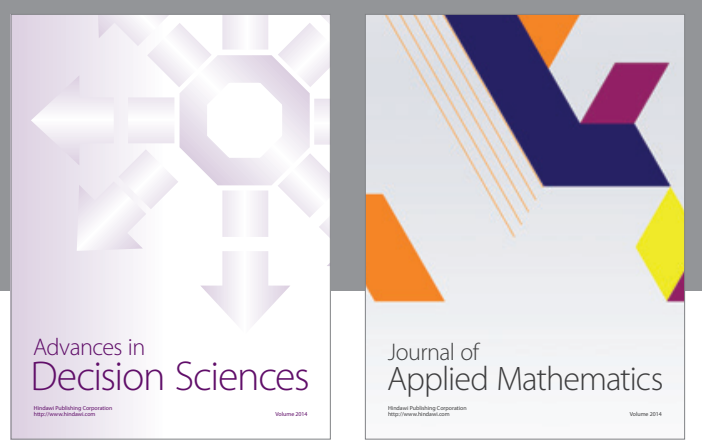

Algebra

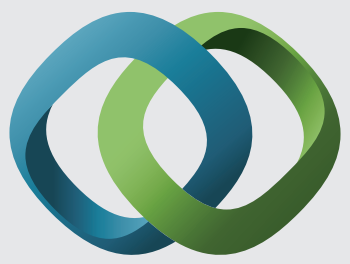

\section{Hindawi}

Submit your manuscripts at

http://www.hindawi.com
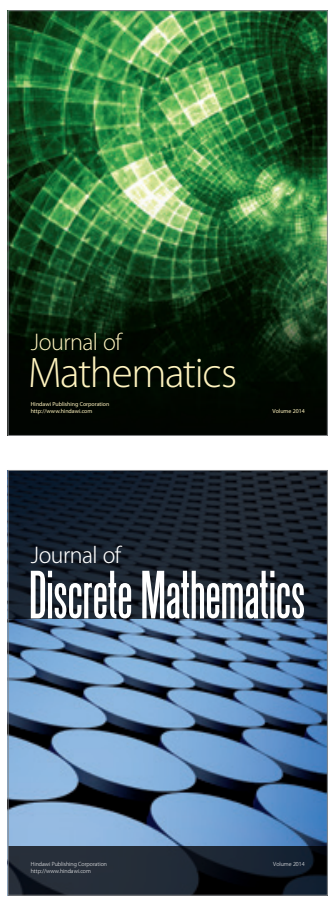

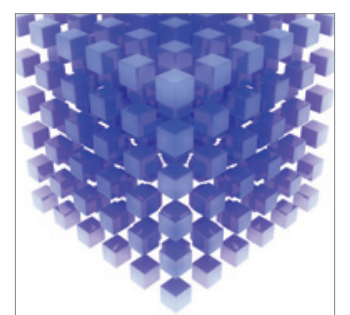

Mathematical Problems in Engineering
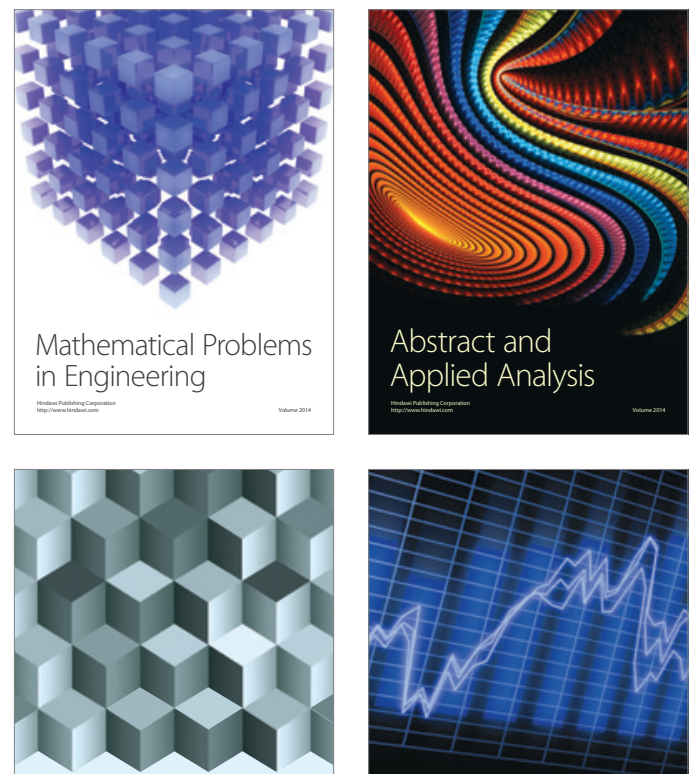

Journal of

Function Spaces

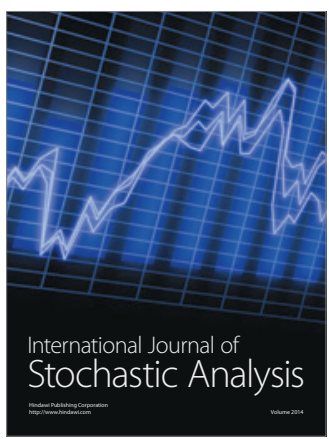

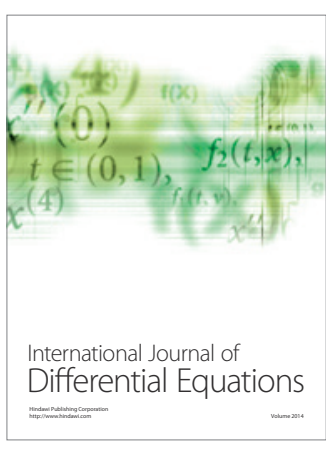
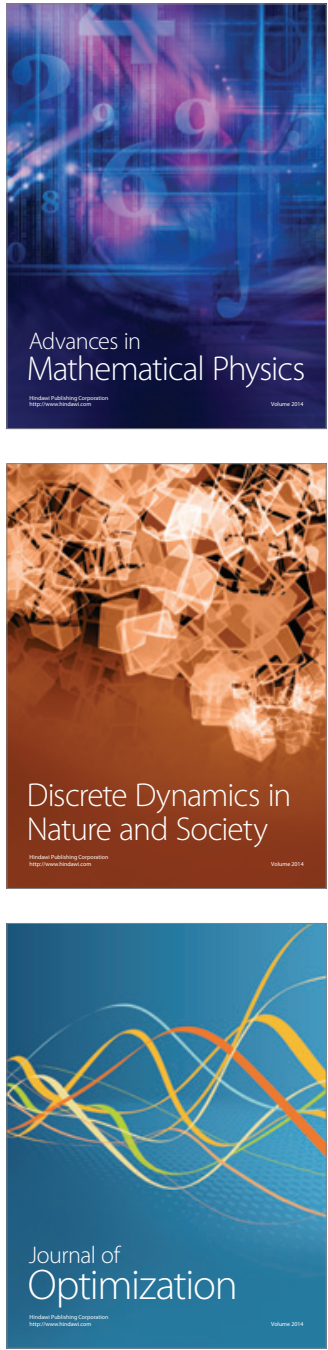